\title{
Thermodynamic fluctuations of electromagnetic field in slightly absorbing media
}

\author{
B.A. Veklenko ${ }^{1}$; Y.B.Sherkunov ${ }^{2 \dagger}$ \\ 1 Institute for High Temperatures RAS, \\ 127412 Moscow, Russia \\ 2 Institute of Thermal Physics of Extremal States \\ of Associated Institute for High Temperatures RAS, \\ 127412 Moscow, Russia
}

Received December 26, 2003, in final form March 10, 2004

\begin{abstract}
A theory of thermodynamic fluctuations of electromagnetic field in slightly absorbing media is developed using the quantum electrodynamics method of $\Gamma$-operators - without phenomenology. The hypothesis offered by Yury L. Klimontovich is under consideration. The necessity of correct consideration of photon-photon correlation functions is shown. The results are compared with the ones obtained with the help of standard theory based upon fluctuation-dissipation theorem (FDT). The latter results are shown to have no field of application at least for the case of thermally excited media of the atoms described with two-level model.
\end{abstract}

Key words: thermodynamic fluctuations, fluctuation-dissipation theorem, correlation function, electromagnetic field in slightly absorbing media

PACS: $42.50 . c t$

\section{Introduction}

Modern theory of electromagnetic fluctuations is a very elegant and important part of theoretical physics. This theory enables us to calculate noise both in electric circuits and optical devices without frequency or wavelength restrictions $[1,2]$. The theory originated both in the Planck formula [3] and Nyquist formula [4]. According to the Planck formula the energy density $u_{\omega}^{0}(T)$ of thermal radiation on a frequency of $\omega$ under temperature $T$ is

$$
u_{\omega}^{0}(T)=\frac{\hbar \omega^{3}}{\pi^{2} c^{3}}\left(\mathrm{e}^{\frac{\hbar \omega}{T}}-1\right)^{-1} .
$$

\footnotetext{
*E-mail: veklenkoba@yandex.ru

†E-mail: sherkunovyb@mail.ru
} 
The history of attempts to extend the Planck formula to the media described by dielectric permittivity is abundant.

The first results were obtained by Clausius [5]. According to Clausius, the extension of equation (1) to the case of transparent media is as follows

$$
u_{\omega}(T)=u_{\omega}^{0}(T) n^{2}(\omega) \frac{\partial \omega n(\omega)}{\partial \omega}
$$

Here, the refractive index $n(\omega)$ is real. The attempts to generalize equation (2) to the case of absorbing media are not completely successful. One should mention here the formulas obtained by Laue and Fragstein which are discussed in monograph [5]. An elegant way of solving the problem was offered by Rytov [1-6]. Since the electromagnetic field is dissipated in an absorbing medium, fluctuating currents $j^{v}(\mathbf{r}, t)$ should exist to maintain the field under the temperature of $T$. The idea of virtual external sources introduced into a fluctuating system is similar to the Langevin idea [7], which was used to investigate Brownian motion. Using the results of paper [8], Rytov supposed that the average value of multiplication of such current densities is

$$
\left\langle j^{v}(\mathbf{r}, t) j^{v^{\prime}}\left(\mathbf{r}^{\prime}, t\right)\right\rangle \sim \delta_{v v^{\prime}}\left(\mathbf{r}-\mathbf{r}^{\prime}\right),
$$

the unknown coefficient of proportionality being the same in all the cases. One can find this coefficient of proportionality by comparing the results of this approach with the solution of a certain model problem obtained using other methods. Since equation (3) is local, it allows us to calculate a fluctuating electromagnetic field in equilibrium for media of any particular configurations [1]. For the case of uniform absorbing medium the following formula was obtained $[1,2]$

$$
\left\langle\mathscr{E}^{v} \mathscr{E}^{v^{\prime}}\right\rangle_{\mathbf{k} \omega}=\mathrm{i}\left(\delta_{\nu \nu^{\prime}}-\frac{k^{\nu} k^{\nu^{\prime}}}{k^{2}}\right) \frac{\hbar \omega^{2} \mathrm{e}^{\frac{\hbar \omega}{T}}}{\mathrm{e}^{\frac{\hbar \omega}{T}}-1}\left(\frac{1}{\omega^{2} \varepsilon(\omega)-c^{2} k^{2}}-\frac{1}{\omega^{2} \varepsilon^{*}(\omega)-c^{2} k^{2}}\right)
$$

where $\varepsilon(\omega)$ is dielectric permittivity, $\mathscr{E}^{v}(\mathbf{r}, t)$ is electric field intensity,

$$
\left\langle\mathscr{E} v \mathscr{E} v^{\prime}\right\rangle_{\mathbf{k} \omega}=\int_{-\infty}^{\infty} \mathrm{e}^{-\mathrm{i} \mathbf{k}\left(\mathbf{r}-\mathbf{r}^{\prime}\right)+\mathrm{i} \omega\left(t-t^{\prime}\right)}\left\langle\mathscr{E} v(\mathbf{r}, t) \mathscr{E}^{\prime}\left(\mathbf{r}^{\prime}, t^{\prime}\right)\right\rangle \mathrm{d}\left(\mathbf{r}-\mathbf{r}^{\prime}\right) \mathrm{d}\left(t-t^{\prime}\right) .
$$

For the purpose of simplicity, we consider transversal electromagnetic field. It is possible that the spectral light fluxes in absorbing media corresponding to equation (4) are significantly different from those in transparent media under the same temperature [9]. The method suggested by Rytov was applied to the theory of molecular forces acting on solid-state bodies by Lifshits [10, 11]. This theory generalized the theory of Casimir and Polder [12]. The next stage of the development of the theory of thermal fluctuations of electromagnetic field is connected with fluctuationdissipation theorem (FDT).

The relation between fluctuation characteristics of point objects and their absorption coefficients is found in original papers $[13,14]$. The theorem was generalized 
to extensive media in $[15,16]$. This generalization results in equation (4), but equation (3) was substantiated more strictly. These results were discussed in the works $[5,17,18]$.

For the case of transparent media, equation (4) transforms to equation (2).

One may put questions: is the description of fluctuating field in real dielectric media with equation (4) adequate enough? Will this formula significantly change if we take into account quantum effects correctly?

In spite the presence of Planck constant in equation (4), the permittivity $\varepsilon(\omega)$, defined as coefficient of proportionality between intensity $\mathscr{E} v$ and displacement $\mathscr{D}^{v}$ of electric field, appears in equation (4) as an attribute of classical physics. In accordance with quantum theory, the quantum average values for $\left\langle\mathscr{E}^{v}\right\rangle$ and $\left\langle\mathscr{D}^{v}\right\rangle$ are equal to zero under the condition of thermodynamic equilibrium. For this reason the definition given above looses its meaning. From quantum point of view it is not evident a priori that $\varepsilon(\omega)$ should appear in equation (4).

Nevertheless, one could think that the equation (4) solves the problem in the framework of classical physics. But in this framework, the presence of the Planck constant in equation (4) seems to be problematic.

There is another more serious objection against equation (4). In his paper [19], Yury Klimontovich suggested a hypothesis, which states that single-time correlation functions of any operators do not depend upon relaxation constants under the condition of thermodynamic equilibrium. According to this hypothesis, the left side of equation (4) integrated over frequency does not depend upon light absorption coefficient in a medium. If we integrate the right side of equation (4) over frequency, we will come to the contradiction with the previous statement (the result of integration will depend upon absorption coefficient).

These reasons inspired us to verify the Klimontovich hypothesis and derive equation (4) using microscopic theory without FDT.

What will microscopic theory give us?

In real media a refractive index is formed as a result of interaction of electromagnetic field with atoms of the media. To describe atoms, one should undoubtedly use quantum theory. How will such a description change the results? It is known that a theory, which deals with quantum description of medium and classical description of electromagnetic field - semiclassical theory - is logically inconsistent [20]. This theory is widely used in practice (e.g. semiclassical laser theory), but one should remember its logical inconsistency and absence of application criteria for the description of fluctuating fields.

What result will we obtain if equation (4) is derived using the consistent quantum electrodynamics?

We will show that a complete solution of the problem for the case of dielectric media described only by standard refractive index is impossible.

If we consider the region of absorption, we mean the region of resonant frequencies of atoms. If these frequencies are energetically significant then we cannot neglect the concentration of excited atoms. In quantum electrodynamics a process of electromagnetic field scattering on excited atoms is described as follows [21]. Firstly, an 
excited atom emits a scattered photon, and then the incident photon is absorbed by the atom. This descriptive image testifies that the processes take place in different moments of time. Since there is an angular correlation between the photons and the photon to be scattered transmits information to the emitted photon, the descriptive image considered above suggests that the process of photon scattering on an excited atom takes a certain area. It means that this process is expected to violate the locale formula (3) even for the case of fluctuating field. Consequently, the violation of equation (4) is expected. Obviously, the descriptive images are not sufficient to modify the theory. This problem is investigated quantitatively below. The expediency of setting up the problem this way is shown.

Let us consider a case of small frequency which is far from resonance. The effect of excited atoms is not significant. Will equations (4) and (2) be correct? We doubt the validity of equations (4) and (2) for the case of transparent media, or, at any rate, it is necessary to indicate their field of application. Quantum electrodynamics points out the other, more delicate thing that complicates the derivation of equations (2) and (4) in this case. If $\hbar \omega \ll T$, the electromagnetic mode of frequency $\omega$ contains - according to Gibbs distribution - a considerable number of quanta, which are described using a single eigenfunction $\varphi$. If a single quantum is scattered the function $\varphi$ describing all the photons is changed. It means that the quanta of a mode cannot be considered mutually independent. While one of the photons is being scattered (it takes some time), the other continue their motion violating the locality of the interaction of the field with the presently unexcited atom. This descriptive image, as shown above, represents the real situation, and in some cases it may violate equations (2) and (4).

It is necessary to mention that in both cases discussed above, we consider the photon correlations. It means that photon correlations should be considered accurately.

In monograph [22], the methods of calculating the electromagnetic fluctuations are considered in quantum electrodynamics framework. The effects we have already mentioned are not found there. All the results of the monograph [22] are obtained using the Matsubara diagram technique. They contain the local refractive index of a medium. What is the matter here? The standard thermodynamic Green function method [22] proves to be not sufficient to describe the correlation processes considered above. To derive a closed system of equations, this standard method requires a discontinuity in the high-order correlation functions of electromagnetic field operators (photon-photon correlation functions) to express them by means of the simplest ones. One cannot strictly estimate what harm such discontinuity does to the theory. To exclude such a questionable operation, we will use hereafter the method which enables us to take into account all the high-order photon-photon correlation functions correctly. Accounting for this dramatically changes the results [23-25], and hence this problem will be considered later.

Finally, we would like to discuss an applicability of FDT. No doubt, this theorem is valid. But the following thing should be mentioned. While deriving the theorem, the existence of Hamiltonian of the system is presupposed. Moreover, the atom en- 
ergy levels are assumed to be infinitely thin. If the exact expression for absorption coefficient is substituted into the right side of the FDT formula, the exact expression for correlation function will be obtained. Really, the expression for absorption coefficient is known in approximation. As an approximation, we can consider the expression in terms of finite atom energy level widths, which is considered in paper [26]. In the frequency regions where absorption is significant, the result obtained using FDT for such an approximation of absorption, coefficient may be very inaccurate. A more elaborate treatment of this problem is given below.

This paper consists of two parts. In the first part, equation (4) is discussed; the internal inconsistency of the standard theory is shown. In the second part, the solution of the problem is suggested.

\section{General principals}

We are interested in the correlation function, given by equation (4). Let us calculate an equivalent function of the vector potentials of electromagnetic field.

$$
\left\langle A^{\nu}(\mathbf{r}, t) A^{\nu^{\prime}}\left(\mathbf{r}^{\prime}, t^{\prime}\right)\right\rangle
$$

Further, we will consider a transverse electromagnetic field. Let this field interact with a gas of nonrelativistic two-level atoms of mass $M$ in quasiresonant way. Quasiresonant interaction implies the following inequality.

$$
\left|\varepsilon_{m}-\varepsilon_{\mu}-k\right| \ll\left|\varepsilon_{m}-\varepsilon_{\mu}\right| .
$$

Here, $\varepsilon_{m}$ is internal energy of excited state of an atom, $\varepsilon_{\mu}$ is the internal energy of its ground state. Let the momenta of atoms obey Maxwell distribution. We suppose that the atoms possess Zeeman sublevels. The parameters $\mathbf{k}$ and $\lambda$ stand for momentum and polarization index $(\lambda=1,2)$ of a photon. By considering two-level atoms, we treat these atoms as principally quantum objects. A passage to the limit $\hbar \rightarrow 0$ is of no meaning to this model. Consequently, we can use the system of units, where $\hbar=c=1$. The Hamiltonian of the system is taken to be

$$
\begin{aligned}
\hat{H} & =\hat{H}^{0}+\hat{H}^{\mathrm{int}}, \quad \hat{H}^{0}=\hat{H}_{a}+\sum_{\mathbf{k} \lambda} \hat{H}_{\mathbf{k} \lambda}^{\mathrm{ph}}, \\
\hat{H}_{a} & =\sum_{\mathbf{p} j} \varepsilon_{j \mathbf{p}} \hat{\beta}_{j \mathbf{p}}^{+} \hat{\beta}_{j \mathbf{p}}, \quad \hat{H}_{\mathbf{k} \lambda}^{\mathrm{ph}}=k \hat{\alpha}_{\mathbf{k} \lambda}^{+} \hat{\alpha}_{\mathbf{k} \lambda}, \quad \varepsilon_{j \mathbf{p}}=\varepsilon_{j}+\frac{p^{2}}{2 M}, \\
\hat{H}^{\mathrm{int}} & =-\int \hat{\psi}^{+}(\mathbf{r}, \mathbf{R}) \hat{\mathbf{P}} \hat{\mathbf{A}} \hat{\psi}(\mathbf{r}, \mathbf{R}) \mathrm{d} \mathbf{r} \mathrm{d} \mathbf{R}, \quad \hat{\mathbf{P}}=-\frac{e}{m} \mathrm{i} \nabla_{\mathrm{r}},
\end{aligned}
$$

where

$$
\begin{aligned}
\hat{\psi}(\mathbf{r}, \mathbf{R}) & =\sum_{\mathbf{p} j} \psi_{j}(\mathbf{r}-\mathbf{R}) \mathrm{e}^{\mathrm{i} \mathbf{p r}} \frac{\hat{\beta}_{j \mathbf{p}}}{\sqrt{V}}, \quad \hat{\mathbf{A}}(\mathbf{r})=\sum_{\mathbf{k} \lambda} \hat{\mathbf{A}}_{\mathbf{k} \lambda}(\mathbf{r}), \\
\hat{\mathbf{A}}_{\mathbf{k} \lambda} & =\frac{\mathbf{e}_{\mathbf{k}}^{\lambda}}{\sqrt{2 k V}}\left(\hat{\alpha}_{\mathbf{k} \lambda} \mathrm{e}^{\mathrm{i} \mathbf{k r}}+\hat{\alpha}_{\mathbf{k} \lambda}^{+} \mathrm{e}^{-\mathbf{i k r}}\right) .
\end{aligned}
$$


The following designations are used here. $\psi_{j}$ is a wave function of a valent electron, $\mathbf{r}$ is a radius-vector of the electron. We will consider the case of atoms with a single valent electron. $\mathbf{R}$ is the radius-vector of the remainder of an atom, $\mathbf{p}$ is its momentum. $\hat{\beta}_{j \mathbf{p}}\left(\hat{\beta}_{j \mathbf{p}}^{+}\right)$denote the operators for annihilation (creation) of atoms in the $(j, \mathbf{p})$ state; $\hat{\alpha}_{\mathbf{k} \lambda}$ and $\hat{\alpha}_{\mathbf{k} \lambda}^{+}$denote the operators for annihilation (creation) of a photon in the $(\mathbf{k}, \lambda)$ mode.

All operators correspond to Bose-Einstein fields. Such a description is justified in the case of temperature nondegenerate gas, which is achieved at sufficiently high temperatures. The unit vectors of linear polarization $\mathrm{e}_{\mathbf{k}}^{\lambda}$ for $\lambda=1 ; 2$ are mutually orthogonal. $V$ is quantization volume, $e$ and $m$ are charge and mass of electron correspondingly. A vacuum term of the Hamiltonian $\hat{H}_{\mathbf{k} \lambda}^{\mathrm{ph}}$ is not significant in our case and is neglected. We will use the following equation of motion

$$
\mathrm{i} \frac{\mathrm{d} \check{L}}{\mathrm{~d} t}=[\check{L} \check{H}]
$$

of field operators $\check{L}(t)$ given in Heisenberg representation. Using equations (5) and (6), we find

$$
\begin{aligned}
\mathrm{i} \frac{\mathrm{d} \check{\alpha}_{\mathbf{k} \lambda}}{\mathrm{d} t}= & k \check{\alpha}_{\mathbf{k} \lambda}-\sum_{m \mu \mathbf{p}^{\prime} \mathbf{p}^{\prime \prime}} \frac{P_{m \mu}^{\lambda *}(\mathbf{k})}{\sqrt{2 k V}} \delta\left(\mathbf{p}^{\prime}, \mathbf{p}^{\prime \prime}-\mathbf{k}\right) \check{\beta}_{\mu \mathbf{p}^{\prime}}^{+} \check{\beta}_{m \mathbf{p}^{\prime \prime}}, \\
\mathrm{i} \frac{\mathrm{d} \check{\alpha}_{\mathbf{k} \lambda}^{+}}{\mathrm{d} t}= & -k \check{\alpha}_{\mathbf{k} \lambda}^{+}+\sum_{m \mu \mathbf{p}^{\prime} \mathbf{p}^{\prime \prime}} \frac{P_{m \mu}^{\lambda}(\mathbf{k})}{\sqrt{2 k V}} \delta\left(\mathbf{p}^{\prime}, \mathbf{k}+\mathbf{p}^{\prime \prime}\right) \check{\beta}_{m \mathbf{p}^{\prime}}^{+} \check{\beta}_{\mu \mathbf{p}^{\prime \prime}}, \\
\mathrm{i} \frac{\mathrm{d} \check{\beta}_{j \mathbf{p}}}{\mathrm{d} t}= & \varepsilon_{j \mathbf{p}} \check{\beta}_{j \mathbf{p}}-\sum_{j^{\prime} \mathbf{p}^{\prime} \mathbf{k} \lambda} \frac{P_{j j^{\prime}}^{\lambda}(\mathbf{k})}{\sqrt{2 k V}} \delta\left(\mathbf{p}, \mathbf{k}+\mathbf{p}^{\prime}\right) \check{\alpha}_{\mathbf{k} \lambda} \check{\beta}_{j^{\prime} \mathbf{p}^{\prime}}-\sum_{j^{\prime} \mathbf{p}^{\prime} \mathbf{k} \lambda} \frac{P_{j^{\prime} j}^{\lambda *}(\mathbf{k})}{V \sqrt{2 k V}} \delta\left(\mathbf{p}, \mathbf{p}^{\prime}-\mathbf{k}\right) \check{\alpha}_{\mathbf{k} \lambda}^{+} \check{\beta}_{j^{\prime} \mathbf{p}^{\prime}}, \\
\mathrm{i} \frac{\mathrm{d} \check{\beta}_{j \mathbf{p}}^{+}}{\mathrm{d} t}= & -\varepsilon_{j \mathbf{p}} \check{\beta}_{j \mathbf{p}}^{+}+\sum_{j^{\prime} \mathbf{p}^{\prime} \mathbf{k} \lambda} \frac{P_{j^{\prime} j}^{\lambda}(\mathbf{k})}{\sqrt{2 k V}} \delta\left(\mathbf{p}, \mathbf{p}^{\prime}-\mathbf{k}\right) \check{\beta}_{j^{\prime} \mathbf{p}^{\prime}}^{+} \check{\alpha}_{\mathbf{k} \lambda} \\
& +\sum_{j^{\prime} \mathbf{p}^{\prime} \mathbf{k} \lambda} \frac{P_{j j^{\prime}}^{\lambda *}(\mathbf{k})}{\sqrt{2 k V}} \delta\left(\mathbf{p}, \mathbf{k}+\mathbf{p}^{\prime}\right) \check{\beta}_{j^{\prime} \mathbf{p}^{\prime}}^{+} \check{\alpha}_{\mathbf{k} \lambda}^{+} .
\end{aligned}
$$

Here

$$
P_{j j^{\prime}}^{\lambda}(\mathbf{k})=\int \psi_{j}^{*}(\boldsymbol{\rho}) \mathbf{e}_{\mathbf{k}}^{\lambda} \hat{\mathbf{P}} \mathrm{e}^{\mathrm{i} \mathbf{k} \boldsymbol{\rho}} \psi_{j^{\prime}}(\boldsymbol{\rho}) \mathrm{d} \boldsymbol{\rho} .
$$

We contrast the subscript $m$ for the Zeeman sublevels of the excited state of atoms with the subscript $\mu$ for the sublevels of the ground state; $j=m, \mu ; \delta\left(\mathbf{p}, \mathbf{p}^{\prime}\right)$ is Kronecker symbol.

\section{Correlation functions of electromagnetic field}

According to equation (6), we can represent the correlation function in question in the following way

$$
\left\langle\check{A}^{\nu}(\mathbf{r}, t) \check{A}^{\nu^{\prime}}\left(\mathbf{r}^{\prime}, t^{\prime}\right)\right\rangle_{\mathbf{k} E}=\int \mathrm{e}^{\mathrm{i} E\left(t-t^{\prime}\right)-\mathrm{i} \mathbf{k}\left(\mathbf{r}-\mathbf{r}^{\prime}\right)}\left\langle\check{A}^{\nu}(\mathbf{r}, t) \check{A}^{\nu^{\prime}}\left(\mathbf{r}^{\prime}, t^{\prime}\right)\right\rangle \mathrm{d}\left(\mathbf{r}-\mathbf{r}^{\prime}\right) \mathrm{d}\left(t-t^{\prime}\right)=
$$




$$
=\frac{1}{2 k}\left(\delta_{\nu \nu^{\prime}}-\frac{k^{\nu} k^{\nu^{\prime}}}{k^{2}}\right)\left(\left\langle\check{\alpha}_{\mathbf{k} \lambda}(t) \check{\alpha}_{\mathbf{k} \lambda}^{+}\left(t^{\prime}\right)\right\rangle_{E}+\left\langle\check{\alpha}_{\mathbf{k} \lambda}^{+}(t) \check{\alpha}_{\mathbf{k} \lambda}\left(t^{\prime}\right)\right\rangle_{E}\right) .
$$

Equations (7) enable us to find an explicit expression for the correlation function $\left\langle\check{\alpha}_{\mathbf{k} \lambda}(t) \check{\alpha}_{\mathbf{k} \lambda}^{+}\left(t^{\prime}\right)\right\rangle \vartheta\left(t-t^{\prime}\right)$. Here $\vartheta$ is the Heaviside function. The brackets $\langle\ldots\rangle$ mean both quantum and statistical averaging over Gibbs distribution. Let us introduce the following designations:

$$
\begin{aligned}
& \vartheta\left(t-t^{\prime}\right)=\vartheta^{>}, \quad \vartheta\left(t^{\prime}-t\right)=\vartheta^{<}, \\
& \left\langle\check{\alpha}_{\mathbf{k} \lambda}(t) \check{\alpha}_{\mathbf{k} \lambda}^{+}\left(t^{\prime}\right)\right\rangle \vartheta\left(t-t^{\prime}\right)=\left\langle\check{\alpha}_{\mathbf{k} \lambda}(t) \check{\alpha}_{\mathbf{k} \lambda}^{+}\left(t^{\prime}\right)\right\rangle^{>}, \\
& \left\langle\check{\alpha}_{\mathbf{k} \lambda}(t) \check{\alpha}_{\mathbf{k} \lambda}^{+}\left(t^{\prime}\right)\right\rangle \vartheta\left(t^{\prime}-t\right)=\left\langle\check{\alpha}_{\mathbf{k} \lambda}(t) \check{\alpha}_{\mathbf{k} \lambda}^{+}\left(t^{\prime}\right)\right\rangle^{<} .
\end{aligned}
$$

In accordance with equation (7)

$$
\begin{aligned}
\left(\mathrm{i} \frac{\mathrm{d}}{\mathrm{d} t}-k\right) & \left\langle\check{\alpha}_{\mathbf{k} \lambda}(t) \check{\alpha}_{\mathbf{k} \lambda}^{+}\left(t^{\prime}\right)\right\rangle=\mathrm{i} \delta\left(t-t^{\prime}\right)\left\langle\hat{\alpha}_{\mathbf{k} \lambda}(t) \hat{\alpha}_{\mathbf{k} \lambda}^{+}\left(t^{\prime}\right)\right\rangle \\
& -\sum_{m \mu \mathbf{p}^{\prime} \mathbf{p}^{\prime \prime}} \frac{P_{m \mu}^{\lambda *}(\mathbf{k})}{\sqrt{2 k V}} \delta\left(\mathbf{p}^{\prime}, \mathbf{p}^{\prime \prime}-\mathbf{k}\right)\left\langle\check{\beta}_{\mu \mathbf{p}^{\prime}}(t)^{+} \check{\beta}_{m \mathbf{p}^{\prime \prime}}(t) \check{\alpha}_{\mathbf{k} \lambda}^{+}\left(t^{\prime}\right)\right\rangle
\end{aligned}
$$

Here

$$
\left\langle\check{\alpha}_{\mathbf{k} \lambda}(t) \check{\alpha}_{\mathbf{k} \lambda}^{+}(t)\right\rangle=\left\langle\hat{\alpha}_{\mathbf{k} \lambda} \hat{\alpha}_{\mathbf{k} \lambda}^{+}\right\rangle .
$$

According to equation (7), the correlation function containing three operators obeys the equation

$$
\begin{aligned}
& \left(\mathrm{i} \frac{\mathrm{d}}{\mathrm{d} t}+\varepsilon_{\mu \mathbf{p}^{\prime}}-\varepsilon_{m \mathbf{p}^{\prime}}\right)\left\langle\check{\beta}_{\mu \mathbf{p}^{\prime}}^{+}(t) \check{\beta}_{m \mathbf{p}^{\prime \prime}}(t) \check{\alpha}_{\mathbf{k} \lambda}^{+}\left(t^{\prime}\right)\right\rangle^{>}= \\
& =\sum_{m_{1} \mathbf{p}_{1} \mathbf{k}_{1} \lambda_{1}} \frac{P_{m_{1} \mu}^{\lambda_{1}}\left(\mathbf{k}_{1}\right)}{\sqrt{2 k_{1} V}} \delta\left(\mathbf{p}_{1}, \mathbf{k}_{1}+\mathbf{p}^{\prime}\right)\left\langle\check{\beta}_{m}^{+} \mathbf{p}_{1}(t) \check{\alpha}_{\mathbf{k}_{1} \lambda_{1}}(t) \check{\beta}_{m \mathbf{p}^{\prime \prime}}(t) \check{\alpha}_{\mathbf{k} \lambda}^{+}\left(t^{\prime}\right)\right\rangle^{>} \\
& \quad-\sum_{\mu_{1} \mathbf{p}_{1} \mathbf{k}_{1} \lambda_{1}} \frac{P_{m \mu_{1}}^{\lambda_{1}}\left(\mathbf{k}_{1}\right)}{\sqrt{2 k_{1} V}} \delta\left(\mathbf{p}_{1}, \mathbf{p}^{\prime \prime}-\mathbf{k}_{1}\right)\left\langle\check{\beta}_{\mu \mathbf{p}^{\prime}}^{+}(t) \check{\alpha}_{\mathbf{k}_{l} \lambda_{l}}(t) \check{\beta}_{\mu_{1} \mathbf{p}_{1}}(t) \check{\alpha}_{\mathbf{k} \lambda}^{+}\left(t^{\prime}\right)\right\rangle^{>}
\end{aligned}
$$

We neglect the terms containing $\check{\alpha}_{\mathbf{k} \lambda}^{+} \check{\alpha}_{\mathbf{k} \lambda}^{+}$and $\check{\alpha}_{\mathbf{k} \lambda} \check{\alpha}_{\mathbf{k} \lambda}$. The contribution of such constructions is too small to be taken into account in our quasiresonance approximation. With the interaction being weak, we can disconnect the correlation functions as follows

$$
\begin{aligned}
\left\langle\check{\beta}_{m l}^{+} \mathbf{p}_{l}(t) \check{\alpha}_{\mathbf{k}_{l} \lambda_{l}}(t) \check{\beta}_{m \mathbf{p}^{\prime \prime}}(t) \check{\alpha}_{\mathbf{k} \lambda}^{+}\left(t^{\prime}\right)\right\rangle^{>} & =\left\langle N_{m \mathbf{p}^{\prime \prime}}\right\rangle\left\langle\check{\alpha}_{\mathbf{k} \lambda}(t) \check{\alpha}_{\mathbf{k} \lambda}^{+}\left(t^{\prime}\right)\right\rangle^{>} \delta_{m_{l} m} \delta_{\mathbf{p}_{l} \mathbf{p}^{\prime \prime}} \delta_{\lambda \lambda_{l}} \delta_{\mathbf{k}_{l} \mathbf{k}}, \\
\left\langle\check{\beta}_{\mu \mathbf{p}^{\prime}}^{+}(t) \check{\beta}_{\mu_{l} \mathbf{p}_{l}} \check{\alpha}_{\mathbf{k}_{l} \lambda_{l}}(t) \check{\alpha}_{\mathbf{k} \lambda}^{+}\left(t^{\prime}\right)\right\rangle & =\left\langle N_{\mu \mathbf{p}^{\prime}}\right\rangle\left\langle\check{\alpha}_{\mathbf{k} \lambda}(t) \check{\alpha}_{\mathbf{k} \lambda}^{+}\left(t^{\prime}\right)\right\rangle^{>} \delta_{\mu_{l} \mu} \delta_{\mathbf{p}_{l} \mathbf{p}^{\prime}} \delta_{\lambda \lambda_{l}} \delta_{\mathbf{k}_{l} \mathbf{k}}, \\
\left\langle\check{\beta}_{\mu \mathbf{p}^{\prime}}^{+}(t) \check{\beta}_{m \mathbf{p}^{\prime \prime}}(t) \check{\alpha}_{\mathbf{k}_{l} \lambda_{l}}(t)\right\rangle & =\left\langle N_{m \mathbf{p}^{\prime}}\right\rangle\left\langle\check{\alpha}_{\mathbf{k} \lambda}(t)\right\rangle \delta_{m \mu} \delta_{\mathbf{p}^{\prime} \mathbf{p}^{\prime \prime}}=0 .
\end{aligned}
$$


Here $\left\langle N_{m \mathbf{p}^{\prime \prime}}\right\rangle=\left\langle\check{\beta}_{m \mathbf{p}^{\prime \prime}}^{+} \check{\beta}_{m \mathbf{p}^{\prime \prime}}\right\rangle,\left\langle N_{\mu \mathbf{p}^{\prime}}\right\rangle=\left\langle\check{\beta}_{\mu \mathbf{p}^{\prime}}^{+} \check{\beta}_{\mu \mathbf{p}^{\prime}}\right\rangle$ are average numbers of the atoms of the gas in the $\left(m, \mathbf{p}^{\prime \prime}\right)$ and $\left(\mu, \mathbf{p}^{\prime}\right)$ states correspondingly. Now, we take into account the fact that all the correlation functions depend upon the difference of time coordinates under the condition of thermodynamic equilibrium. Using Fourier transformation

$$
\left\langle\check{\alpha}_{\mathbf{k} \lambda}(t) \check{\alpha}_{\mathbf{k} \lambda}^{+}\left(t^{\prime}\right)\right\rangle_{E}^{>}=\int_{-\infty}^{\infty} \mathrm{e}^{\mathrm{i} E\left(t-t^{\prime}\right)}\left\langle\check{\alpha}_{\mathbf{k} \lambda}(t) \check{\alpha}_{\mathbf{k} \lambda}^{+}\left(t^{\prime}\right)\right\rangle^{>} \mathrm{d}\left(t-t^{\prime}\right)
$$

and equations (9), (10), we find

$$
\left\langle\check{\alpha}_{\mathbf{k} \lambda}(t) \check{\alpha}_{\mathbf{k} \lambda}^{+}\left(t^{\prime}\right)\right\rangle_{E}^{>}=\frac{\mathrm{i}\left\langle\hat{\alpha}_{\mathbf{k} \lambda} \hat{\alpha}_{\mathbf{k} \lambda}^{+}\right\rangle}{\operatorname{En}(E)-k} .
$$

The function

$$
n(E)=1-\frac{1}{E} \sum_{m \mu \mathbf{p}^{\prime} \mathbf{p}^{\prime \prime}}\left|\frac{P_{m \mu}^{\lambda}(\mathbf{k})}{\sqrt{2 k V}}\right|^{2} \frac{\delta\left(\mathbf{p}^{\prime}, \mathbf{p}^{\prime \prime}-\mathbf{k}\right)}{E-\varepsilon_{m \mathbf{p}^{\prime \prime}}+\varepsilon_{\mu \mathbf{p}^{\prime}}+\mathrm{i} 0}\left(\left\langle N_{\mu \mathbf{p}^{\prime}}\right\rangle-\left\langle N_{m \mathbf{p}^{\prime \prime}}\right\rangle\right)
$$

is known to be a complex refractive index of a medium.

This function is obtained by way of correlation discontinuity. Thus it is valid for rare-field media

$$
n_{0} \lambda^{3} \frac{\gamma_{\mathrm{r}}}{\gamma_{\mathrm{D}}}<1, \quad \lambda=\frac{2 \pi}{k} .
$$

This inequality signifies that the second term of the right side of equation (12) is small in comparison with the first one.

Here $n_{0}$ denotes total concentration of atoms; $\gamma_{\mathrm{r}}, \gamma_{\mathrm{D}}$ are radiation and Doppler spectral line widths of radiation.

The following formulas are obtained in the same way

$$
\begin{aligned}
\left\langle\check{\alpha}_{\mathbf{k} \lambda}(t) \check{\alpha}_{\mathbf{k} \lambda}^{+}\left(t^{\prime}\right)\right\rangle_{E}^{<} & =\frac{-\mathrm{i}\left\langle\hat{\alpha}_{\mathbf{k} \lambda} \hat{\alpha}_{\mathbf{k} \lambda}^{+}\right\rangle}{E n^{*}(E)-k}, \\
\left\langle\check{\alpha}_{\mathbf{k} \lambda}^{+}\left(t^{\prime}\right) \check{\alpha}_{\mathbf{k} \lambda}(t)\right\rangle_{E}^{>} & =\frac{\mathrm{i}\left\langle\hat{\alpha}_{\mathbf{k} \lambda}^{+} \hat{\alpha}_{\mathbf{k} \lambda}\right\rangle}{E n(E)-k}, \\
\left\langle\check{\alpha}_{\mathbf{k} \lambda}\left(t^{\prime}\right) \check{\alpha}_{\mathbf{k} \lambda}^{+}(t)\right\rangle_{E}^{<} & =\frac{-\mathrm{i}\left\langle\hat{\alpha}_{\mathbf{k} \lambda}^{+} \hat{\alpha}_{\mathbf{k} \lambda}\right\rangle}{E n^{*}(E)-k} .
\end{aligned}
$$

Further, we will use the following symmetry equations

$$
\begin{array}{ll}
\left\langle\check{\alpha}_{\mathbf{k} \lambda}\left(t^{\prime}\right) \check{\alpha}_{\mathbf{k} \lambda}^{+}(t)\right\rangle_{E}^{>}=\left\langle\check{\alpha}_{\mathbf{k} \lambda}(t) \check{\alpha}_{\mathbf{k} \lambda}^{+}\left(t^{\prime}\right)\right\rangle_{-E}^{<}, & \left\langle\check{\alpha}_{\mathbf{k} \lambda}\left(t^{\prime}\right) \check{\alpha}_{\mathbf{k} \lambda}^{+}(t)\right\rangle_{E}^{<}=\left\langle\check{\alpha}_{\mathbf{k} \lambda}(t) \check{\alpha}_{\mathbf{k} \lambda}^{+}\left(t^{\prime}\right)\right\rangle_{-E}^{>}, \\
\left\langle\check{\alpha}_{\mathbf{k} \lambda}^{+}(t) \check{\alpha}_{\mathbf{k} \lambda}\left(t^{\prime}\right)\right\rangle_{E}^{>}=\left\langle\check{\alpha}_{\mathbf{k} \lambda}^{+}\left(t^{\prime}\right) \check{\alpha}_{\mathbf{k} \lambda}(t)\right\rangle_{-E}^{<}, & \left\langle\check{\alpha}_{\mathbf{k} \lambda}^{+}(t) \check{\alpha}_{\mathbf{k} \lambda}\left(t^{\prime}\right)\right\rangle_{E}^{<}=\left\langle\check{\alpha}_{\mathbf{k} \lambda}^{+}\left(t^{\prime}\right) \check{\alpha}_{\mathbf{k} \lambda}(t)\right\rangle_{-E}^{>} .
\end{array}
$$

Equations (11)-(12), (14)-(15) are not sufficient to find the correlation functions of electromagnetic field operators, since the right sides of the equations contain unknown quantities. To solve the problem, one can use FDT. 


\section{Fluctuation-dissipation theorem}

The fluctuation-dissipation theorem establishes the equalities between the correlation and the Green functions of the system under the condition of thermodynamic equilibrium $[13-15,18,26]$. The form of these equalities does not depend upon the presence of interaction in the system described by a complete Hamiltonian. It means that the equalities are of the same form for the case of free systems. But it is simple to find the equalities for free systems. Let us consider a free electromagnetic field.

$$
\begin{aligned}
\check{\alpha}_{\mathbf{k} \lambda}(t) & =\hat{\alpha}_{\mathbf{k} \lambda} \mathrm{e}^{-\mathrm{i} k t}, \quad \check{\alpha}_{\mathbf{k} \lambda}^{+}(t)=\hat{\alpha}_{\mathbf{k} \lambda}^{+} \mathrm{e}^{\mathrm{i} k t}, \\
{\left[\hat{\alpha}_{\mathbf{k} \lambda} \hat{\alpha}_{\mathbf{k} \lambda}^{+}\right] } & =\hat{\alpha}_{\mathbf{k} \lambda} \hat{\alpha}_{\mathbf{k} \lambda}^{+}-\hat{\alpha}_{\mathbf{k} \lambda}^{+} \hat{\alpha}_{\mathbf{k} \lambda}=\delta_{k k^{\prime}} \delta_{\lambda \lambda^{\prime}} .
\end{aligned}
$$

A direct verification gives the following results for Fourier transforms

$$
\begin{gathered}
\left\langle\check{\alpha}_{\mathbf{k} \lambda}(t) \check{\alpha}_{\mathbf{k} \lambda}^{+}\left(t^{\prime}\right)\right\rangle_{E}=2 \pi \delta(E-k)\left\langle\hat{\alpha}_{\mathbf{k} \lambda} \hat{\alpha}_{\mathbf{k} \lambda}^{+}\right\rangle, \\
\left\langle\check{\alpha}_{\mathbf{k} \lambda}^{+}(t) \check{\alpha}_{\mathbf{k} \lambda}\left(t^{\prime}\right)\right\rangle_{E}=2 \pi \delta(E+k)\left\langle\hat{\alpha}_{\mathbf{k} \lambda}^{+} \hat{\alpha}_{\mathbf{k} \lambda}\right\rangle, \\
\left\langle\check{\alpha}_{\mathbf{k} \lambda}(t) \check{\alpha}_{\mathbf{k} \lambda}^{+}\left(t^{\prime}\right)\right\rangle_{E}=\left\langle\check{\alpha}_{\mathbf{k} \lambda}(t) \check{\alpha}_{\mathbf{k} \lambda}^{+}\left(t^{\prime}\right)\right\rangle_{E}^{>}+\left\langle\check{\alpha}_{\mathbf{k} \lambda}(t) \check{\alpha}_{\mathbf{k} \lambda}^{+}\left(t^{\prime}\right)\right\rangle_{E}^{<}= \\
=\frac{\mathrm{e}^{E / T}}{\mathrm{e}^{E / T}-1}\left(D_{\mathrm{r}}\left(t-t^{\prime}\right)-D_{a}\left(t-t^{\prime}\right)\right), \\
\left\langle\check{\alpha}_{\mathbf{k} \lambda}^{+}\left(t^{\prime}\right) \check{\alpha}_{\mathbf{k} \lambda}(t)\right\rangle_{E}=\left\langle\check{\alpha}_{\mathbf{k} \lambda}^{+}\left(t^{\prime}\right) \check{\alpha}_{\mathbf{k} \lambda}(t)\right\rangle_{E}^{>}+\left\langle\check{\alpha}_{\mathbf{k} \lambda}^{+}\left(t^{\prime}\right) \check{\alpha}_{\mathbf{k} \lambda}(t)\right\rangle_{E}^{<}= \\
=\frac{1}{e^{E / T}-1}\left(D_{\mathrm{r}}\left(t-t^{\prime}\right)-D_{a}\left(t-t^{\prime}\right)\right),
\end{gathered}
$$

where

$$
D_{\mathrm{r}}\left(t-t^{\prime}\right)=\left\langle\left[\check{\alpha}_{\mathbf{k} \lambda}(t) \check{\alpha}_{\mathbf{k} \lambda}^{+}\left(t^{\prime}\right)\right]\right\rangle_{E}^{>}, \quad D_{a}\left(t-t^{\prime}\right)=\left\langle\left[\check{\alpha}_{\mathbf{k} \lambda}(t) \check{\alpha}_{\mathbf{k} \lambda}^{+}\left(t^{\prime}\right)\right]\right\rangle_{E}^{<} .
$$

We take into account that

$$
\left\langle\hat{\alpha}_{\mathbf{k} \lambda} \hat{\alpha}_{\mathbf{k} \lambda}^{+}\right\rangle=\frac{e^{k / T}}{e^{k / T}-1}, \quad\left\langle\hat{\alpha}_{\mathbf{k} \lambda}^{+} \hat{\alpha}_{\mathbf{k} \lambda}\right\rangle=\frac{1}{e^{k / T}-1} .
$$

The fluctuation-dissipation theorem states that equations (16) and (17) are valid for the case of any interactions in the system, which are described by a certain Hamiltonian of interaction [27]. Using equations (14)-(17) we arrive at

$$
\begin{aligned}
\left\langle\check{\alpha}_{\mathbf{k} \lambda}(t) \check{\alpha}_{\mathbf{k} \lambda}^{+}\left(t^{\prime}\right)\right\rangle_{E} & =\mathrm{i}\left(\frac{1}{E n(E)-k}-\frac{1}{E n^{*}(E)-k}\right) \frac{e^{E / T}}{e^{E / T}-1}, \\
\left\langle\check{\alpha}_{\mathbf{k} \lambda}\left(t^{\prime}\right) \check{\alpha}_{\mathbf{k} \lambda}^{+}(t)\right\rangle_{E} & =-\mathrm{i}\left(\frac{1}{E n^{*}(-E)+k}-\frac{1}{E n(-E)+k}\right) \frac{1}{e^{E / T}-1}, \\
\left\langle\check{\alpha}_{\mathbf{k} \lambda}^{+}\left(t^{\prime}\right) \check{\alpha}_{\mathbf{k} \lambda}(t)\right\rangle_{E} & =\mathrm{i}\left(\frac{1}{E n(E)-k}-\frac{1}{E n^{*}(E)-k}\right) \frac{1}{e^{E / T}-1}, \\
\left\langle\check{\alpha}_{\mathbf{k} \lambda}^{+}(t) \check{\alpha}_{\mathbf{k} \lambda}\left(t^{\prime}\right)\right\rangle_{E} & =-\mathrm{i}\left(\frac{1}{E n^{*}(-E)+k}-\frac{1}{E n(-E)+k}\right) \frac{e^{E / T}}{e^{E / T}-1} .
\end{aligned}
$$


Substituting equations (18)-(21) into (8), we find

$$
\begin{aligned}
\left\langle\check{A}^{\nu}(\mathbf{r}, t) \check{A}^{\nu^{\prime}}\left(\mathbf{r}^{\prime}, t^{\prime}\right)\right\rangle_{\mathbf{k} E}=\frac{\mathrm{i}}{2 k}\left(\delta_{\nu \nu^{\prime}}-\frac{k^{\nu} k^{\nu^{\prime}}}{k^{2}}\right) \frac{e^{E / T}}{e^{E / T}-1} \\
\quad \times\left(\frac{1}{E n(E)-k}-\frac{1}{E n^{*}(E)-k}-\frac{1}{E n^{*}(-E)+k}+\frac{1}{E n(-E)+k}\right) .
\end{aligned}
$$

In the quasiresonant approximation, the function $n(E)$ differs from unity only in the neighborhood of $E \approx \varepsilon_{m}-\varepsilon_{\mu}>0$. Thus, the following substitution does not affect the accuracy of calculations.

$n(E) \rightarrow n(E) \vartheta(E)+n^{*}(-E) \vartheta(-E), \quad n^{*}(-E) \rightarrow n^{*}(-E) \vartheta(-E)+n(E) \vartheta(E)$.

Now, we obtain

$$
\begin{aligned}
& \left\langle\check{A}^{\nu}(\mathbf{r}, t) \check{A}^{\nu^{\prime}}\left(\mathbf{r}^{\prime}, t^{\prime}\right)\right\rangle_{\mathbf{k} E}= \\
& \quad=\mathrm{i}\left(\delta_{\nu \nu^{\prime}}-\frac{k^{\nu} k^{\nu^{\prime}}}{k^{2}}\right) \frac{e^{E / T}}{e^{E / T}-1}\left(\frac{1}{E^{2} \varepsilon(E)-k^{2}}-\frac{1}{E^{2} \varepsilon^{*}(E)-k^{2}}\right)
\end{aligned}
$$

where

$$
\varepsilon(E)=\left(n(E) \vartheta(E)+n^{*}(-E) \vartheta(-E)\right)^{2}
$$

is dielectric permittivity of the medium. Equation (22) is equivalent to equation (4).

Now, we will show that this formula is not valid in general case. Equation (22) is a direct consequence of equations (18)-(21). But these equations contradict the consequences of equations (11), (14). Equations (11), (14) result in

$$
\left\langle\check{\alpha}_{\mathbf{k} \lambda}(t) \check{\alpha}_{\mathbf{k} \lambda}^{+}\right\rangle_{E}=\mathrm{i}\left\langle\hat{\alpha}_{\mathbf{k} \lambda} \hat{\alpha}_{\mathbf{k} \lambda}^{+}\right\rangle\left(\frac{1}{E n(E)-k}-\frac{1}{E n^{*}(E)-k}\right) .
$$

The left sides of equations (18) and (23) coincide. Consequently, the right sides of the equations should coincide as well.

$$
\begin{aligned}
& \mathrm{i}\left(\frac{1}{E n(E)-k}-\frac{1}{E n^{*}(E)-k}\right) \frac{e^{E / T}}{e^{E / T}-1}= \\
& \quad=\mathrm{i}\left\langle\hat{\alpha}_{\mathbf{k} \lambda} \hat{\alpha}_{\mathbf{k} \lambda}^{+}\right\rangle\left(\frac{1}{E n(E)-k}-\frac{1}{E n^{*}(E)-k}\right) .
\end{aligned}
$$

However, in general case, such an equality is impossible. After cancelling equation (24) by a monomial factor we get the equality of functions depending on different arguments. This contradiction signifies that the correlation function $\left\langle\check{\alpha}_{\mathbf{k} \lambda}(t) \check{\alpha}_{\mathbf{k} \lambda}^{+}\left(t^{\prime}\right)\right\rangle_{E}$, which is calculated using FDT and approximate $D_{\mathrm{r}}$ and $D_{a}$, is suspicious. According to equation (18)

$$
\left\langle\hat{\alpha}_{\mathbf{k} \lambda} \hat{\alpha}_{\mathbf{k} \lambda}^{+}\right\rangle=\mathrm{i} \int_{-\infty}^{\infty} \frac{e^{E / T}}{e^{E / T}-1}\left(\frac{1}{E n(E)-k}-\frac{1}{E n^{*}(E)-k}\right) \frac{\mathrm{d} E}{2 \pi} .
$$


First, let us consider transparent media and real-valued $n(E)$

$$
\frac{1}{E n(E)-k}-\frac{1}{E n^{*}(E)-k} \rightarrow-2 \pi \mathrm{i} \delta(E n(E)-k) .
$$

Strictly speaking, there are no absolutely transparent media. It results from the fact that a real-valued $n(E)$, which describes such media, does not obey the dispersion relation [15], i.e. causality principle. The case of transparent media is a conditional one. Namely, one should consider a function $\delta(E n(E)-k)$, which appears for the case of transparent media, as the one possessing the following properties

$$
\int_{-\infty}^{\infty} \delta(E n(E)-k) \mathrm{d} E=1, \quad \int_{-\infty}^{\infty} \delta(E n(E)-k) \mathrm{d} k=1
$$

The former equality implies the identity

$$
\int_{-\infty}^{\infty}\left(\frac{1}{E n(E)-k}-\frac{1}{E n^{*}(E)-k}\right) \frac{\mathrm{d} E}{2 \pi}=-\mathrm{i}\left\langle\left[\check{\alpha}_{\mathbf{k} \lambda}(t) \check{\alpha}_{\mathbf{k} \lambda}^{+}(t)\right]\right\rangle=-\mathrm{i} .
$$

For the case of transparent media, we can rewrite equation (24)

$$
\frac{e^{E / T}}{e^{E / T}-1} \delta(E n(E)-k)=\int_{-\infty}^{\infty} \frac{e^{E^{\prime} / T}}{e^{E^{\prime} / T}-1} \delta\left(E^{\prime} n\left(E^{\prime}\right)-k\right) \mathrm{d} E^{\prime} \delta(E n(E)-k) .
$$

This equation is evidently valid for the case of electromagnetic field in a vacuum, where $n=1$. Both sides of the equation contain generalized function $\delta(E-k)$, which cannot be cancelled. The right and left sides of the equation differ from zero only if $E=k$. For the case of transparent media, the equation is valid only under the conditions described by equations (26).

If the media are absorbing and $n(E)$ is complex, the violation of equation (24) in the region of absorption frequencies is evident.

Since the first relation of equations (26) is obviously conditional, the situations appear, where equation (24) is violated even for the case of transparent media. We will consider these situations in detail below.

The point is that the exact FDT representing an equality of generalized functions is proved at the assumption that the complete system is described by a certain Hamiltonian. In other words, there are no energy (frequency) relaxation broadenings in the system. Consequently, both sides of equation (24) contain generalized functions, which cannot be cancelled. If we use an approximation by introducing relaxation broadenings - we cannot make other approximations - the fluctuationdissipation theorem, as shown above, results in contradictory expressions. In other words, FDT is not stable to approximations. This problem is widely discussed in the following papers $[28,29,31]$. To obtain a result without contradictions we should use equation (23), where the levels of approximation of both sides are adjusted, 
and take into account equation (27). Here, all the improvements are connected only with explicit expression for $n(E)$ under the condition of equation (27). Below, we will show that the single-time correlation function of equation (23), as it results from Klimontovich hypothesis, does not depend upon the absorption coefficient. If a result of calculations obtained in the approximation used here with the help of FDT or other methods cannot be represented as equation (23) with the condition equation (27), such a result is contradictory. It is the situation, we face analyzing equation (18). This is the reason why equation (22) and standard equation (4) are contradictory. This contradiction is most distinct for the region of the frequencies of absorption.

Let us mention the following thing. Equation (25), as it is shown below, results from equation (17), which has no field of application if $D_{\mathrm{r}}$ are $D_{a}$ are approximate. But, if we substitute equation (25) into (23), we will get the following formula.

$$
\begin{aligned}
\left\langle\check{\alpha}_{\mathbf{k} \lambda}(t) \check{\alpha}_{\mathbf{k} \lambda}^{+}\left(t^{\prime}\right)\right\rangle_{E} & =-\left(\frac{1}{E n(E)-k}-\frac{1}{E n^{*}(E)-k}\right) \\
& \times \int_{-\infty}^{\infty} \frac{e^{E^{\prime} / T}}{e^{E^{\prime} / T}-1}\left(\frac{1}{E^{\prime} n\left(E^{\prime}\right)-k}-\frac{1}{E^{\prime} n^{*}\left(E^{\prime}\right)-k}\right) \frac{\mathrm{d} E^{\prime}}{2 \pi} .
\end{aligned}
$$

This formula instead of equation (17) is valid for the situations, where the concentrations of excited atoms may be neglected and the single-time correlation functions are described with standard refractive index $n(E)$. We will prove the validity of equation (28) below.

Now, the problem is to use equation (23) to analyze the field correlation functions and to derive the expression for $\left\langle\hat{\alpha}_{\mathbf{k} \lambda} \hat{\alpha}_{\mathbf{k} \lambda}^{+}\right\rangle$without FDT.

To calculate $\left\langle\hat{\alpha}_{\mathbf{k} \lambda} \hat{\alpha}_{\mathbf{k} \lambda}^{+}\right\rangle$, we use the $\Gamma$-operator method [23]. This method has some advantages of the temperature Green function method. Besides, it makes it possible to take into account the photon-photon correlation functions in all orders. The method enables us to calculate the single-time correlation functions without FDT and, as a result, to solve the problem. The single-time correlation functions give equilibrium wavelength distributions of photons, including the regions of absorption.

Here, we return once again to Klimontovich hypothesis to adjust it. Let us consider the problem of evolution of electromagnetic field in the media of two-level atoms under a certain temperature $T$. Let us divide the processes of interaction of electromagnetic field with the atoms into two groups. The first, coherent group includes the processes of elastic scattering when the atoms do not change their quantum state (including translational). The second, incoherent group, includes the processes when the initial state of atoms is changed (spontaneous radiation, Raman scattering, and induced radiation processes). The term coherent channel signifies the series of coherent processes only. In the other case, the channel is incoherent.

One of the authors proved the theorem [23], which states that the incoherent channel does not contribute to a single-time correlation function under the conditions of thermodynamic equilibrium and Gibbs distribution. This theorem results in a significant consequence. First of all, we would like to mention that when any 
kinetic equation of Boltsman type is derived, the incoherent channel plays the main part. Consequently, in accordance with the theorem, the single-time correlation functions of dynamic quantities do not depend upon relaxation constants defined by the equations of Boltsman type.

Thus, the Klimontovich hypothesis is formulated in an extra strict manner. Really, the results may contain the relaxation constants determining the decays of the coherent channel. But, the coefficient of absorption, which is equal to imaginary part of complex refractive index, does not belong to this permitted group of relaxation constants.

It is evident that if we found an exact result for any single-time correlation function, e.g.

$$
\left\langle\hat{\alpha}_{\mathbf{k} \lambda}^{+} \hat{\alpha}_{\mathbf{k} \lambda}\right\rangle=S p \mathrm{e}^{-\hat{H} / T} \hat{\alpha}_{\mathbf{k} \lambda}^{+} \hat{\alpha}_{\mathbf{k} \lambda},
$$

it would contain no relaxation constants at all. But here we discuss the problem as to which relaxation constants can or cannot be consequently obtained, if we use approximations.

The calculated single-time correlation function of electromagnetic potentials, as one of the authors has shown [23], does not contain the refractive index, as in equations (18)-(21). It contains another function named casual refractive index [32]. The wavelength distribution of the photons obtained by one of the authors [23] does not solve the problem of frequency distribution. Equation (23) seems to solve the problem. But the second factor of equation (23) was calculated without correct accounting for photon-photon correlation functions. Since these correlation functions significantly change the first factor of equation (23), we should investigate the effect of these functions on the second factor.

Hereinafter we calculate the second factor of equation (23) using the $\Gamma$-operator method taking into account all the orders of photon-photon correlation functions.

\section{Method of split $\Gamma$-operators}

Here, we give the basic concepts of $\Gamma$-operator method.

Let a number of photons in a $(\mathbf{k}, \lambda)$ mode be $N_{\mathbf{k} \lambda}$. We introduce an auxiliary $\Gamma$-space with a creation vector $\rangle_{\Gamma}^{0}$. Creation operators $\hat{\mathscr{A}}^{+}\left(N_{\mathbf{k} \lambda}\right)$ are defined in the $\Gamma$-space so that a wave function of the $N_{\mathrm{k} \lambda}$ photon state is $\left.\hat{\mathscr{A}}^{+}\left(N_{\mathbf{k} \lambda}\right)\right\rangle_{\Gamma}^{0}$. Annihilation operators $\hat{\mathscr{A}}\left(N_{\mathrm{k} \lambda}\right)$ are defined correspondingly. Since the degrees of the operators $\hat{\mathscr{A}}\left(N_{\mathrm{k} \lambda}\right)$ and $\hat{\mathscr{A}}^{+}\left(N_{\mathbf{k} \lambda}\right)$ do not exceed unity, we can choose any commutation relations. Let us accept the following relation

$$
\left[\hat{\mathscr{A}}\left(N_{\mathbf{k} \lambda}\right) \hat{\mathscr{A}}^{+}\left(N_{\mathbf{k}^{\prime} \lambda^{\prime}}^{\prime}\right)\right]=\delta_{\lambda \lambda^{\prime}} \delta_{\mathbf{k k}^{\prime}} \delta\left(N_{\mathbf{k} \lambda}, N_{\mathbf{k} \lambda}^{\prime}\right) .
$$

A basis wave function of a free electromagnetic field in the standard space is

$$
\prod_{\mathbf{k} \lambda} \varphi\left(N_{\mathbf{k} \lambda} \mid \zeta_{\mathbf{k} \lambda}\right)
$$


Here, $\varphi\left(N_{\mathbf{k} \lambda} \mid \zeta_{\mathbf{k} \lambda}\right)$ is a wave function of quantum oscillator. Here, we will not discuss the physical meaning of $\zeta_{\mathbf{k} \lambda}$.

Equation (29) corresponds to the following formula in $\Gamma$-space

$$
\left.\prod_{\mathbf{k} \lambda} \hat{\mathscr{A}}^{+}\left(N_{\mathbf{k} \lambda}\right)\right\rangle_{\Gamma}^{0}
$$

To get equation (30) from equation (29) we used a unitary operator [23]

$$
\left.\hat{O}=\prod_{\mathbf{k} \lambda} \hat{\Phi}^{+}\left(\zeta_{\mathbf{k} \lambda}\right)\right\rangle_{\Gamma}^{0}, \quad \hat{\Phi}\left(\zeta_{\mathbf{k} \lambda}\right)=\sum_{N_{\mathbf{k} \lambda}} \hat{\mathscr{A}}\left(N_{\mathbf{k} \lambda}\right) \varphi\left(N_{\mathbf{k} \lambda} \mid \zeta_{\mathbf{k} \lambda}\right)
$$

It is evident that

$$
\int \hat{\Phi}^{+}\left(\zeta_{\mathbf{k} \lambda}\right) \hat{\Phi}\left(\zeta_{\mathbf{k} \lambda}\right) \mathrm{d} \zeta_{\mathbf{k} \lambda}=\sum_{N_{\mathbf{k} \lambda}} \hat{\mathscr{A}}^{+}\left(N_{\mathbf{k} \lambda}\right) \hat{\mathscr{A}}\left(N_{\mathbf{k} \lambda}\right)=1
$$

Using equation (31), we obtain the following expression for the Hamiltonian in the $\Gamma$-space out of equations (5)-(6).

$$
\begin{aligned}
\hat{H}_{\Gamma}= & \hat{H}_{a}+\sum_{\mathbf{k} \lambda} \int \hat{\Phi}^{+}\left(\zeta_{\mathbf{k} \lambda}\right) \hat{H}_{\mathbf{k} \lambda}^{\mathrm{ph}} \hat{\Phi}\left(\zeta_{\mathbf{k} \lambda}\right) \mathrm{d} \zeta_{\mathbf{k} \lambda} \\
& -\sum_{\mathbf{k} \lambda} \int \hat{\Phi}^{+}\left(\zeta_{\mathbf{k} \lambda}\right) \hat{\psi}^{+}(\mathbf{r}, \mathbf{R}) \hat{\mathbf{P}} \hat{\mathbf{A}}_{\mathbf{k} \lambda} \hat{\psi}(\mathbf{r}, \mathbf{R}) \hat{\Phi}\left(\zeta_{\mathbf{k} \lambda}\right) \mathrm{d} \zeta_{\mathbf{k} \lambda} \mathrm{d} \mathbf{R} \mathrm{d} \mathbf{r}
\end{aligned}
$$

$\mathrm{Or}$

$$
\begin{aligned}
\hat{H}_{\Gamma}= & \hat{H}_{a}+\sum_{N_{\mathbf{k} \lambda}} \sum_{\mathbf{k} \lambda} \hat{\mathscr{A}}^{+}\left(N_{\mathbf{k} \lambda}\right) \varepsilon\left(N_{\mathbf{k} \lambda}\right) \hat{\mathscr{A}}\left(N_{\mathbf{k} \lambda}\right) \\
& -\sum_{N_{\mathbf{k} \lambda}, N_{\mathbf{k} \lambda}^{\prime}} \sum_{\mathbf{k} \lambda} \int \hat{\mathscr{A}}^{+}\left(N_{\mathbf{k} \lambda}\right) \hat{\psi}^{+}(\mathbf{r}, \mathbf{R}) \hat{\mathbf{P}} \mathbf{A}_{\mathbf{k} \lambda}\left(N_{\mathbf{k} \lambda} N_{\mathbf{k} \lambda}^{\prime}\right) \hat{\psi}(\mathbf{r}, \mathbf{R}) \hat{\mathscr{A}}\left(N_{\mathbf{k} \lambda}^{\prime}\right) \mathrm{d} \mathbf{R} \mathrm{d} \mathbf{r}
\end{aligned}
$$

where

$$
\mathbf{A}_{\mathbf{k} \lambda}\left(N_{\mathbf{k} \lambda}, N_{\mathbf{k} \lambda}^{\prime}\right)=\int \varphi\left(N_{\mathbf{k} \lambda} \mid \zeta_{\mathbf{k} \lambda}\right) \hat{\mathbf{A}}_{\mathbf{k} \lambda} \varphi\left(N_{\mathbf{k} \lambda}^{\prime} \mid \zeta_{\mathbf{k} \lambda}\right) \mathrm{d} \zeta_{\mathbf{k} \lambda}, \quad \varepsilon\left(N_{\mathbf{k} \lambda}\right)=k N_{\mathbf{k} \lambda} .
$$

In Heisenberg representation

$$
\check{\mathscr{A}}\left(N_{\mathbf{k} \lambda}, t\right)=\mathrm{e}^{\mathrm{i} \hat{H}_{\Gamma} t} \hat{\mathscr{A}}\left(N_{\mathbf{k} \lambda}\right) \mathrm{e}^{-\mathrm{i} \hat{H}_{\Gamma} t}, \quad \check{\mathscr{A}}^{+}\left(N_{\mathbf{k} \lambda}, t\right)=\mathrm{e}^{\mathrm{i} \hat{H}_{\Gamma} t} \hat{\mathscr{A}}^{+}\left(N_{\mathbf{k} \lambda}\right) \mathrm{e}^{-\mathrm{i} \hat{H}_{\Gamma} t} .
$$

The equations of motion are as follows

$$
\mathrm{i} \frac{\mathrm{d}}{\mathrm{d} t} \check{\mathscr{A}}\left(N_{\mathbf{k} \lambda}, t\right)=\left[\check{\mathscr{A}}\left(N_{\mathbf{k} \lambda}, t\right), \hat{H}_{\Gamma}\right], \quad \mathrm{i} \frac{\mathrm{d}}{\mathrm{d} t} \check{\mathscr{A}}^{+}\left(N_{\mathbf{k} \lambda}, t\right)=\left[\check{\mathscr{A}}^{+}\left(N_{\mathbf{k} \lambda}, t\right), \hat{H}_{\Gamma}\right] .
$$

After simple calculations, we obtain

$$
\begin{aligned}
\mathrm{i} \frac{\mathrm{d}}{\mathrm{d} t} \check{\mathscr{A}}\left(N_{\mathbf{k} \lambda}, t\right)=\varepsilon\left(N_{\mathbf{k} \lambda}\right) \check{\mathscr{A}}\left(N_{\mathbf{k} \lambda}, t\right) \\
\quad-\sum_{N_{\mathbf{k} \lambda}^{\prime}} \int \check{\psi}^{+}(\mathbf{r}, \mathbf{R}) \hat{\mathbf{P}} \mathbf{A}_{\mathbf{k} \lambda}\left(N_{\mathbf{k} \lambda}, N_{\mathbf{k} \lambda}^{\prime}\right) \check{\psi}(\mathbf{r}, \mathbf{R}) \check{\mathscr{A}}\left(N_{\mathbf{k} \lambda}^{\prime}, t\right) \mathrm{d} \mathbf{R} \mathrm{d} \mathbf{r},
\end{aligned}
$$




$$
\begin{aligned}
-\mathrm{i} \frac{\mathrm{d}}{\mathrm{d} t} \check{\mathscr{A}}^{+}\left(N_{\mathbf{k} \lambda}, t\right)=\varepsilon\left(N_{\mathbf{k} \lambda}\right) \check{\mathscr{A}}^{+}\left(N_{\mathbf{k} \lambda}, t\right) \\
\quad-\sum_{N_{\mathbf{k} \lambda}^{\prime}} \check{\mathscr{A}}^{+}\left(N_{\mathbf{k} \lambda}^{\prime}, t\right) \int \check{\psi}^{+}(\mathbf{r}, \mathbf{R}) \hat{\mathbf{P}} \mathbf{A}_{\mathbf{k} \lambda}\left(N_{\mathbf{k} \lambda}^{\prime}, N_{\mathbf{k} \lambda}\right) \check{\psi}(\mathbf{r}, \mathbf{R}) \mathrm{d} \mathbf{R} \mathrm{d} \mathbf{r},
\end{aligned}
$$

\section{Correlation function $\left\langle\check{\alpha}_{\mathrm{k} \lambda}(t) \check{\alpha}_{\mathrm{k} \lambda}^{+}\left(t^{\prime}\right)\right\rangle$ in $\Gamma$-space}

It is evident that the results of calculations of correlation functions do not depend upon the representation we use. We will represent the correlation functions in question using operators $\hat{\mathscr{A}}$ and $\hat{\mathscr{A}}^{+}$. According to the definition

$$
\left\langle\check{\alpha}_{\mathbf{k} \lambda}(t) \check{\alpha}_{\mathbf{k} \lambda}^{+}\left(t^{\prime}\right)\right\rangle=\left\langle\mathrm{e}^{\mathrm{i} \hat{H} t} \hat{\alpha}_{\mathbf{k} \lambda} \mathrm{e}^{-\hat{H} t} \mathrm{e}^{\mathrm{i} \hat{H} t^{\prime}} \hat{\alpha}_{\mathbf{k} \lambda}^{+} \mathrm{e}^{-\mathrm{i} \hat{H} t^{\prime}}\right\rangle .
$$

The result does not change if we replace all the operators and wave functions by those rewritten in the $\Gamma$-representation.

$$
\left\langle\check{\alpha}_{\mathbf{k} \lambda}(t) \check{\alpha}_{\mathbf{k} \lambda}^{+}\left(t^{\prime}\right)\right\rangle=\left\langle\mathrm{e}^{\mathrm{i} \hat{H}_{\Gamma} t} \hat{\alpha}_{\mathbf{k} \lambda}^{(\Gamma)} \mathrm{e}^{-\hat{H}_{\Gamma} t} \mathrm{e}^{\mathrm{i} \hat{H}_{\Gamma} t^{\prime}} \hat{\alpha}_{\mathbf{k} \lambda}^{(\Gamma)+} \mathrm{e}^{-\mathrm{i} \hat{H}_{\Gamma} t^{\prime}}\right\rangle_{\Gamma} .
$$

Here, we use averaging over Gibbs distribution and quantum states in $\Gamma$-space.

$$
\begin{aligned}
\hat{\alpha}_{\mathbf{k} \lambda}^{(\Gamma)} & =\hat{O} \hat{\alpha}_{\mathbf{k} \lambda} \hat{O}^{+}=\sum_{N_{\mathbf{k} \lambda}, N_{\mathbf{k} \lambda}^{\prime}} \hat{\mathscr{A}}^{+}\left(N_{\mathbf{k} \lambda}\right) \alpha_{\mathbf{k} \lambda}\left(N_{\mathbf{k} \lambda}, N_{\mathbf{k} \lambda}^{\prime}\right) \hat{\mathscr{A}}\left(N_{\mathbf{k} \lambda}^{\prime}\right), \\
\hat{\alpha}_{\mathbf{k} \lambda}^{(\Gamma)+} & =\hat{O} \hat{\alpha}_{\mathbf{k} \lambda}^{+} \hat{O}^{+}=\sum_{N_{\mathbf{k} \lambda}, N_{\mathbf{k} \lambda}^{\prime}} \hat{\mathscr{A}}^{+}\left(N_{\mathbf{k} \lambda}\right) \alpha_{\mathbf{k} \lambda}^{+}\left(N_{\mathbf{k} \lambda}, N_{\mathbf{k} \lambda}^{\prime}\right) \hat{\mathscr{A}}\left(N_{\mathbf{k} \lambda}^{\prime}\right),
\end{aligned}
$$

where

$$
\begin{aligned}
\alpha_{\mathbf{k} \lambda}\left(N_{\mathbf{k} \lambda}, N_{\mathbf{k} \lambda}^{\prime}\right) & =\int \varphi\left(N_{\mathbf{k} \lambda} \mid \zeta_{\mathbf{k} \lambda}\right) \hat{\alpha}_{\mathbf{k} \lambda} \varphi\left(N_{\mathbf{k} \lambda}^{\prime} \mid \zeta_{\mathbf{k} \lambda}\right) \mathrm{d} \zeta_{\mathbf{k} \lambda}=\sqrt{N_{\mathbf{k} \lambda}^{\prime}} \delta\left(N_{\mathbf{k} \lambda}, N_{\mathbf{k} \lambda}^{\prime}-1\right), \\
\alpha_{\mathbf{k} \lambda}^{+}\left(N_{\mathbf{k} \lambda}, N_{\mathbf{k} \lambda}^{\prime}\right) & =\int \varphi\left(N_{\mathbf{k} \lambda} \mid \zeta_{\mathbf{k} \lambda}\right) \hat{\alpha}_{\mathbf{k} \lambda}^{+} \varphi\left(N_{\mathbf{k} \lambda}^{\prime} \mid \zeta_{\mathbf{k} \lambda}\right) \mathrm{d} \zeta_{\mathbf{k} \lambda}=\sqrt{N_{\mathbf{k} \lambda}^{\prime}+1} \delta\left(N_{\mathbf{k} \lambda}, N_{\mathbf{k} \lambda}^{\prime}+1\right) .
\end{aligned}
$$

Substituting equation (35) into equation (34) we get

$$
\begin{aligned}
& \left\langle\check{\alpha}_{\mathbf{k} \lambda}(t) \check{\alpha}_{\mathbf{k} \lambda}^{+}\left(t^{\prime}\right)\right\rangle=\sum\left\langle\check{\mathscr{A}}^{+}\left(N_{\mathbf{k} \lambda}, t\right) \alpha_{\mathbf{k} \lambda}\left(N_{\mathbf{k} \lambda}, N_{\mathbf{k} \lambda}^{(1)}\right)\right. \\
& \left.\quad \times \check{\mathscr{A}}\left(N_{\mathbf{k} \lambda}^{(1)}, t\right) \check{\mathscr{A}}^{+}\left(N_{\mathbf{k} \lambda}^{(2)}, t^{\prime}\right) \alpha_{\mathbf{k} \lambda}^{+}\left(N_{\mathbf{k} \lambda}^{(2)}, N_{\mathbf{k} \lambda}^{(3)}\right) \check{\mathscr{A}}\left(N_{\mathbf{k} \lambda}^{(3)}, t^{\prime}\right)\right\rangle_{\Gamma} .
\end{aligned}
$$

Here, we sum over all $N_{\mathbf{k} \lambda}, N_{\mathbf{k} \lambda}^{(1)}, N_{\mathbf{k} \lambda}^{(2)}$, and $N_{\mathbf{k} \lambda}^{(3)}$.

Now, to calculate the correlation function in question we should find the following correlation function

$$
\left\langle\check{\mathscr{A}}^{+}\left(N_{\mathbf{k} \lambda}, t\right) \check{\mathscr{A}}\left(N_{\mathbf{k} \lambda}^{(1)}, t\right) \check{\mathscr{A}}^{+}\left(N_{\mathbf{k} \lambda}^{(2)}, t^{\prime}\right) \check{\mathscr{A}}\left(N_{\mathbf{k} \lambda}^{(3)}, t^{\prime}\right)\right\rangle_{\Gamma} .
$$




\section{Equation of motion for $\left\langle\check{\mathscr{A}}^{+} \check{\mathscr{A}} \check{\mathscr{A}}+\check{\mathscr{A}}\right\rangle_{\Gamma}$}

To calculate correlation function given by equation (38), we should write the equation for this function. To simplify the equation, we omit subscripts $\mathbf{k}, \lambda$. Acting on the left-side of equation (38) with the operator $\mathrm{i}(\mathrm{d} / \mathrm{d} t)$ and taking into account equations (32), (33), we obtain

$$
\begin{aligned}
\left(\mathrm{i} \frac{\mathrm{d}}{\mathrm{d} t}\right. & \left.+k N-k N^{\prime}\right)\left\langle\check{\mathscr{A}}^{+}(N, t) \check{\mathscr{A}}\left(N^{\prime}, t\right) \ldots\right\rangle_{\Gamma}^{\rangle}=\mathrm{i} \delta(t)\left\langle\check{\mathscr{A}}^{+}(N) \check{\mathscr{A}}\left(N^{\prime}\right) \ldots\right\rangle_{\Gamma} \\
& +\sum_{N^{\prime \prime}}\left\langle\check { \mathscr { A } } ^ { + } ( N ^ { \prime \prime } , t ) \sum _ { m \mu \mathbf { p } \mathbf { p } ^ { \prime } } \left[\frac{P_{m \mu}^{\lambda}(\mathbf{k})}{\sqrt{2 k V}} \delta\left(\mathbf{p}, \mathbf{k}+\mathbf{p}^{\prime}\right) \check{\beta}_{m \mathbf{p}}^{+}(t) \alpha\left(N^{\prime \prime}, N\right) \check{\beta}_{\mu \mathbf{p}^{\prime}}(t)\right.\right. \\
& \left.\left.+\frac{P_{m \mu}^{\lambda *}(\mathbf{k})}{\sqrt{2 k V}} \sigma \delta\left(\mathbf{p}, \mathbf{p}^{\prime}-\mathbf{k}\right) \check{\beta}_{\mu \mathbf{p}}^{+}(t) \alpha^{+}\left(N^{\prime \prime}, N\right) \check{\beta}_{m \mathbf{p}^{\prime}}(t)\right] \check{\mathscr{A}}\left(N^{\prime}, t\right) \ldots\right\rangle_{\Gamma}^{>} \\
& -\sum_{N^{\prime \prime}}\left\langle\check { \mathscr { A } } ^ { + } ( N , t ) \sum _ { m \mu \mathbf { p } \mathbf { p } ^ { \prime } } \left[\frac{P_{m \mu}^{\lambda}(\mathbf{k})}{\sqrt{2 k V}} \delta\left(\mathbf{p}, \mathbf{k}+\mathbf{p}^{\prime}\right) \check{\beta}_{m \mathbf{p}}^{+}(t) \alpha\left(N^{\prime}, N^{\prime \prime}\right) \check{\beta}_{\mu \mathbf{p}^{\prime}}(t)\right.\right. \\
& \left.\left.+\frac{P_{m \mu}^{\lambda *}(\mathbf{k})}{\sqrt{2 k V}} \delta\left(\mathbf{p}, \mathbf{p}^{\prime}-\mathbf{k}\right) \check{\beta}_{\mu \mathbf{p}}^{+}(t) \alpha^{+}\left(N^{\prime}, N^{\prime \prime}\right) \check{\beta}_{m \mathbf{p}^{\prime}}(t)\right] \check{\mathscr{A}}\left(N^{\prime \prime}, t\right) \ldots\right\rangle_{\Gamma}^{>}
\end{aligned}
$$

Here $t^{\prime}=0$.

Now, we should write the equations for the operators of right-side of equation (39). According to equation (7), it is sufficient to use the following equations for the operators $\check{\beta}_{i \mathbf{p}}$ and $\check{\beta}_{i \mathbf{p}}^{+}$

$$
\mathrm{i} \frac{\mathrm{d} \check{\beta}_{i \mathbf{p}}}{\mathrm{d} t}=\varepsilon_{i \mathbf{p}} \check{\beta}_{i \mathbf{p}}, \quad-\mathrm{i} \frac{\mathrm{d} \check{\beta}_{i \mathbf{p}}^{+}}{\mathrm{d} t}=\varepsilon_{j \mathbf{p}} \check{\beta}_{i \mathbf{p}}^{+} .
$$

Such a simplification is justified because the last terms of right sides of equation (7) contain operators $\check{\alpha}_{\mathbf{k} \lambda}$ and $\check{\alpha}_{\mathbf{k} \lambda}^{+}$. When we turn to the $\Gamma$-space, we use the operators given by equations (35) instead of $\check{\alpha}_{\mathbf{k} \lambda}$ and $\check{\alpha}_{\mathbf{k} \lambda}^{+}$. Eventually, it results in the multiplications of two annihilation operators

$$
\check{\mathscr{A}}\left(N_{\mathbf{k} \lambda}, t\right) \check{\mathscr{A}}\left(N_{\mathbf{k}^{\prime} \lambda^{\prime}}^{\prime}, t\right) .
$$

We should put the terms of this kind to zero. If $(\mathbf{k}, \lambda)$ is equal to $\left(\mathbf{k}^{\prime}, \lambda^{\prime}\right)$, the operators $\breve{\mathscr{A}}\left(N_{\mathbf{k} \lambda}, t\right) \check{\mathscr{A}}\left(N_{\mathbf{k} \lambda}^{\prime}, t\right)$ acting on any wave function of physical state result in zero [23,24]. If $(\mathbf{k}, \lambda)$ is not equal to $\left(\mathbf{k}^{\prime}, \lambda^{\prime}\right)$, we come to $\left\langle\hat{\alpha}_{\mathbf{k} \lambda}\right\rangle=\left\langle\hat{\alpha}_{\mathbf{k} \lambda}^{+}\right\rangle=0$ after the procedure of disconnecting the correlation functions we use here.

As a result, we obtain four new correlation functions in the right side of equation (39). The equation for the first of them is

$$
\left(\mathrm{i} \frac{\mathrm{d}}{\mathrm{d} t}-k N^{\prime}+k N^{\prime \prime}-\varepsilon_{\mu \mathbf{p}^{\prime}}+\varepsilon_{m \mathbf{p}}\right)\left\langle\check{\mathscr{A}}^{+}\left(N^{\prime \prime}, t\right) \check{\beta}_{m \mathbf{p}}^{+}(t) \check{\beta}_{\mu \mathbf{p}^{\prime}}(t) \check{\mathscr{A}}\left(N^{\prime}, t\right) \ldots\right\rangle_{\Gamma}^{>}=
$$




$$
\begin{aligned}
= & \sum_{N_{1} m_{1} \mu_{1} \mathbf{p}_{1} \mathbf{p}_{1}^{\prime}} \frac{P_{m_{1} \mu_{1}}^{\lambda *}(\mathbf{k})}{\sqrt{2 k V}} \delta\left(\mathbf{p}_{1}, \mathbf{p}_{1}^{\prime}-\mathbf{k}\right) \\
& \times\left\langle\check{\mathscr{A}}^{+}\left(N_{1}, t\right) \check{\beta}_{\mu_{1} \mathbf{p}_{1}}^{+}(t) \check{\beta}_{m_{1} \mathbf{p}_{1}^{\prime}}(t) \check{\beta}_{m \mathbf{p}}^{+}(t) \check{\beta}_{\mu \mathbf{p}^{\prime}}(t) \alpha^{+}\left(N_{1}, N^{\prime \prime}\right) \check{\mathscr{A}}\left(N^{\prime}, t\right) \ldots\right\rangle_{\Gamma}^{>} \\
& -\sum_{N_{1} m_{1} \mu_{1} \mathbf{p}_{1} \mathbf{p}_{1}^{\prime}} \frac{P_{m_{1} \mu_{1}}^{\lambda}(\mathbf{k})}{\sqrt{2 k V}} \delta\left(\mathbf{p}_{1}, \mathbf{k}+\mathbf{p}_{1}^{\prime}\right) \\
& \times\left\langle\check{\mathscr{A}}^{+}\left(N^{\prime \prime}, t\right) \check{\beta}_{m \mathbf{p}}^{+}(t) \check{\beta}_{\mu \mathbf{p}^{\prime}}(t) \check{\beta}_{\mu_{1} \mathbf{p}_{1}}^{+}(t) \check{\beta}_{m_{1} \mathbf{p}_{1}^{\prime}}(t) \alpha\left(N^{\prime}, N_{1}\right) \check{\mathscr{A}}\left(N_{1}, t\right) \ldots\right\rangle_{\Gamma}^{>}
\end{aligned}
$$

Now, we can disconnect the correlation functions of the right side of equation (40), isolating the atomic correlation functions. In such an approximation, the correlation functions of the right side of equation (40) are

$$
\begin{aligned}
& \left\langle N_{\mu \mathbf{p}^{\prime}}\right\rangle \delta_{m m_{1}} \delta_{\mu \mu_{1}} \delta_{\mathbf{p p}_{1}^{\prime}} \delta_{\mathbf{p}^{\prime} \mathbf{p}_{1}}\left\langle\check{\mathscr{A}}^{+}\left(N_{1}, t\right) \check{\mathscr{A}}\left(N^{\prime}, t\right) \ldots\right\rangle_{\Gamma}^{\rangle}, \\
& \left\langle N_{m \mathbf{p}}\right\rangle \delta_{m m_{1}} \delta_{\mu \mu_{1}} \delta_{\mathbf{p p}_{1}^{\prime}} \delta_{\mathbf{p}^{\prime} \mathbf{p}_{1}}\left\langle\check{\mathscr{A}}^{+}\left(N^{\prime \prime}, t\right) \check{\mathscr{A}}\left(N_{1}, t\right) \ldots\right\rangle_{\Gamma}^{>},
\end{aligned}
$$

where $\left\langle N_{\mu \mathbf{p}^{\prime}}\right\rangle=\left\langle\check{\beta}_{\mu \mathbf{p}^{\prime}}^{+} \check{\beta}_{\mu \mathbf{p}^{\prime}}\right\rangle_{\Gamma},\left\langle N_{m \mathbf{p}}\right\rangle=\left\langle\check{\beta}_{m \mathbf{p}}^{+} \check{\beta}_{m \mathbf{p}}\right\rangle_{\Gamma}$ are the numbers of atoms in the states $\left(\mu, \mathbf{p}^{\prime}\right)$ and $(m, \mathbf{p})$.

Taking Fourier transformation and using equations (40) and (36), we obtain

$$
\begin{aligned}
\left\langle\check{\mathscr{A}}\left(N^{\prime \prime}, t\right) \check{\beta}_{m \mathbf{p}}^{+}(t) \check{\beta}_{\mu \mathbf{p}^{\prime}}(t) \check{\mathscr{A}}\left(N^{\prime}, t\right) \ldots\right\rangle_{\Gamma E}^{>}=\frac{1}{E-k N^{\prime}+k N^{\prime \prime}-\varepsilon_{\mu \mathbf{p}^{\prime}}+\varepsilon_{m \mathbf{p}}+\mathrm{i} 0} \\
\times\left(\frac{P_{m \mu}^{\lambda *}(\mathbf{k})}{\sqrt{2 k V}} \delta\left(\mathbf{p}^{\prime}, \mathbf{p}-\mathbf{k}\right)\left\langle N_{\mu \mathbf{p}^{\prime}}\right\rangle \sqrt{N^{\prime \prime}+1}\left\langle\check{\mathscr{A}}^{+}\left(N^{\prime \prime}+1, t\right) \check{\mathscr{A}}\left(N^{\prime}, t\right) \ldots\right\rangle_{\Gamma E}^{>}\right. \\
\left.-\frac{P_{m \mu}^{\lambda}(\mathbf{k})}{\sqrt{2 k V}} \delta\left(\mathbf{p}^{\prime}, \mathbf{k}+\mathbf{p}\right)\left\langle N_{m \mathbf{p}}\right\rangle \sqrt{N^{\prime}+1}\left\langle\check{\mathscr{A}}^{+}\left(N^{\prime \prime}, t\right) \check{\mathscr{A}}\left(N^{\prime}+1, t\right) \ldots\right\rangle_{\Gamma E}^{>}\right) .
\end{aligned}
$$

The terms i0 in denominators are explained by analyticity requirements in the upper semiplane of complex $E$.

We calculated the other correlation functions of the right side of equation (39).

Let us return to equation (37). Taking into account the explicite expressions for matrix elements of $\alpha_{\mathbf{k} \lambda}, \alpha_{\mathbf{k} \lambda}^{+}$, we obtain

$$
\begin{aligned}
& \left\langle\check{\alpha}_{\mathbf{k} \lambda}(t) \check{\alpha}_{\mathbf{k} \lambda}^{+}\left(t^{\prime}\right)\right\rangle= \\
& \quad=\sum_{N N_{1}} \sqrt{N+1} \sqrt{N_{1}}\left\langle\check{\mathscr{A}}^{+}(N, t) \check{\mathscr{A}}(N+1, t) \check{\mathscr{A}}^{+}\left(N_{1}, 0\right) \check{\mathscr{A}}^{\prime}\left(N_{1}-1,0\right)\right\rangle_{\Gamma}^{>} .
\end{aligned}
$$

Thus, we should find the correlation function

$$
\left\langle\check{\mathscr{A}}^{+}(N, t) \check{\mathscr{A}}(N+1, t) \check{\mathscr{A}}^{+}\left(N^{(1)}, 0\right) \check{\mathscr{A}}\left(N^{(1)}-1,0\right)\right\rangle_{\Gamma}^{\rangle} .
$$


Putting $N^{\prime}=N+1$ in equation (39) and using the procedure resulting in equation (41), we obtain the following equation for the Fourier transforms

$$
\begin{aligned}
& (E-k)\left\langle\check{\mathscr{A}}^{+}(N, t) \check{\mathscr{A}}(N+1, t) \ldots\right\rangle_{\Gamma E}^{>}=\mathrm{i}\left\langle\check{\mathscr{A}}^{+}(N) \check{\mathscr{A}}(N+1)\right\rangle_{\Gamma} \\
& +\sum_{m \mu \mathbf{p} \mathbf{p}^{\prime}}\left|\frac{P_{m \mu}^{\lambda}(\mathbf{k})}{\sqrt{2 k V}}\right|^{2}\left\{\left[\frac{N \delta\left(\mathbf{p}, \mathbf{k}+\mathbf{p}^{\prime}\right)\left\langle N_{\mu \mathbf{p}^{\prime}}\right\rangle}{E-2 k+\varepsilon_{m \mathbf{p}}-\varepsilon_{\mu \mathbf{p}^{\prime}}+\mathrm{i} 0}+\frac{(N+1) \delta\left(\mathbf{p}, \mathbf{k}+\mathbf{p}^{\prime}\right)\left\langle N_{m \mathbf{p}}\right\rangle}{E-\varepsilon_{m \mathbf{p}}+\varepsilon_{\mu \mathbf{p}^{\prime}}+\mathrm{i} 0}\right.\right. \\
& \left.+\frac{(N+2) \delta\left(\mathbf{p}, \mathbf{k}+\mathbf{p}^{\prime}\right)\left\langle N_{m \mathbf{p}}\right\rangle}{E-2 k+\varepsilon_{m \mathbf{p}}-\varepsilon_{\mu \mathbf{p}^{\prime}}+\mathrm{i} 0}+\frac{(N+1) \delta\left(\mathbf{p}, \mathbf{k}+\mathbf{p}^{\prime}\right)\left\langle N_{m \mathbf{p}}\right\rangle}{E-\varepsilon_{m \mathbf{p}}+\varepsilon_{\mu \mathbf{p}^{\prime}}+\mathrm{i} 0}\right] \\
& \times\left\langle\check{\mathscr{A}}^{+}(N, t) \check{\mathscr{A}}(N+1, t) \ldots\right\rangle_{\Gamma E}^{>} \\
& -\left[\frac{\sqrt{N(N+1)} \delta\left(\mathbf{p}, \mathbf{k}+\mathbf{p}^{\prime}\right)\left\langle N_{m \mathbf{p}}\right\rangle}{E-2 k+\varepsilon_{m \mathbf{p}}-\varepsilon_{\mu \mathbf{p}^{\prime}}+\mathrm{i} 0}+\frac{\sqrt{N(N+1)} \delta\left(\mathbf{p}, \mathbf{k}+\mathbf{p}^{\prime}\right)\left\langle N_{m \mathbf{p}}\right\rangle}{E-\varepsilon_{m \mathbf{p}}+\varepsilon_{\mu \mathbf{p}^{\prime}}+\mathrm{i} 0}\right] \\
& \times\left\langle\check{\mathscr{A}}^{+}(N-1, t) \check{\mathscr{A}}(N, t) \ldots\right\rangle_{\Gamma E}^{>} \\
& -\left[\frac{\sqrt{(N+2)(N+1)} \delta\left(\mathbf{p}, \mathbf{k}+\mathbf{p}^{\prime}\right)\left\langle N_{\mu \mathbf{p}^{\prime}}\right\rangle}{E-2 k+\varepsilon_{m \mathbf{p}}-\varepsilon_{\mu \mathbf{p}^{\prime}}+\mathrm{i} 0}+\frac{\sqrt{(N+2)(N+1)} \delta\left(\mathbf{p}, \mathbf{k}+\mathbf{p}^{\prime}\right)\left\langle N_{\mu \mathbf{p}}\right\rangle}{E-\varepsilon_{m \mathbf{p}}+\varepsilon_{\mu \mathbf{p}^{\prime}}+\mathrm{i} 0}\right] \\
& \left.\times\left\langle\check{\mathscr{A}}^{+}(N+1, t) \check{\mathscr{A}}(N+2, t) \ldots\right\rangle_{\Gamma E}^{>}\right\} \text {. }
\end{aligned}
$$

Now we should multiply both sides of this equation by $\sqrt{(N+1) N_{1}}$ according to equation (42) and sum the equation over $0<N, N_{1}<\infty$. After cancelling the like terms, we get

$$
\begin{aligned}
(E-k)\left\langle\check{\alpha} \check{\alpha}^{+}\right. & \rangle_{E}^{>}=\mathrm{i}\left\langle\hat{\alpha} \hat{\alpha}^{+}\right\rangle \\
& +\sum_{m \mu \mathbf{p} \mathbf{p}^{\prime}}\left|\frac{P_{m \mu}^{\lambda}(\mathbf{k})}{\sqrt{2 k V}}\right|^{2} \frac{\left\langle N_{\mu \mathbf{p}^{\prime}}\right\rangle-\left\langle N_{m \mathbf{p}}\right\rangle}{E-\varepsilon_{m \mathbf{p}}+\varepsilon_{\mu \mathbf{p}^{\prime}}+\mathrm{i} 0} \delta\left(\mathbf{p}, \mathbf{k}+\mathbf{p}^{\prime}\right)\left\langle\check{\alpha} \check{\alpha}^{+}\right\rangle_{E}^{>}
\end{aligned}
$$

The solution of this equation coincides with equation (11). Equations (14) are restored in the same way.

Thus, we have shown that a correct accounting for the photon-photon correlation functions does not affect the second factor of the right side of equation (23) in the approximation given by equation (13), while it significantly changes the first factor.

Thus, we can find the correlation function (8) in our approximation using equations (11) and (14). But the single-time correlation function $\left\langle\hat{\alpha}_{\mathbf{k} \lambda}^{+} \hat{\alpha}_{\mathbf{k} \lambda}\right\rangle$ is still unknown. 


\section{Correlation function $\left\langle\hat{\alpha}_{\mathrm{k} \lambda}^{+} \hat{\alpha}_{\mathrm{k} \lambda}\right\rangle$}

One of the authors obtained the following result [23]

$$
\left\langle\hat{\alpha}_{\mathbf{k} \lambda}^{+} \hat{\alpha}_{\mathbf{k} \lambda}\right\rangle=-\frac{1}{Z} \sum_{N_{\mathbf{k} \lambda}=1}^{\infty} N_{\mathbf{k} \lambda} \Delta\left(\frac{1}{T}, N_{\mathbf{k} \lambda}\right)
$$

here $Z$ is a normalizing constant. The Laplace transform

$$
\Delta\left(s, N_{\mathbf{k} \lambda}\right)=\int_{0}^{\infty} \mathrm{e}^{-\tau s} \Delta\left(\tau, N_{\mathbf{k} \lambda}\right) \mathrm{d} \tau
$$

is found to be

$$
\Delta\left(s, N_{\mathbf{k} \lambda}\right)=\frac{\Delta^{0}\left(s, N_{\mathbf{k} \lambda}\right)}{1-\Delta^{0}\left(s, N_{\mathbf{k} \lambda}\right) \mathscr{P}\left(s, N_{\mathbf{k} \lambda}\right)}=\frac{1}{s+k N_{\mathbf{k} \lambda}-\mathscr{P}\left(s, N_{\mathbf{k} \lambda}\right)},
$$

where

$$
\Delta^{0}(s)=-\frac{1}{s+k N_{\mathbf{k} \lambda}}
$$

In approximation (13)

$$
\mathscr{P}\left(s, N_{\mathbf{k} \lambda}\right)=\left(N_{\mathbf{k} \lambda}+1\right) \alpha^{\mathbf{k} \lambda}(s)+N_{\mathbf{k} \lambda} c^{\mathbf{k} \lambda}(s),
$$

where

$$
\begin{aligned}
& c^{\mathbf{k} \lambda}(s)=-\sum_{m \mu \mathbf{p}}\left|\frac{P_{m \mu}^{\lambda}(\mathbf{k})}{\sqrt{2 k V}}\right|^{2} \frac{\left\langle N_{\mu \mathbf{p}}\right\rangle}{s+\varepsilon_{m}(\mathbf{p}+\mathbf{k})-\varepsilon_{\mu}(\mathbf{p})+k\left(N_{\mathbf{k} \lambda}-1\right)}, \\
& a^{\mathbf{k} \lambda}(s)=-\sum_{m \mu \mathbf{p}}\left|\frac{P_{m \mu}^{\lambda}(\mathbf{k})}{\sqrt{2 k V}}\right|^{2} \frac{\left\langle N_{m \mathbf{p}}\right\rangle}{s+\varepsilon_{\mu}(\mathbf{p}-\mathbf{k})-\varepsilon_{m}(\mathbf{p})+k\left(N_{\mathbf{k} \lambda}+1\right)} .
\end{aligned}
$$

The inverse transformation returns

$$
\Delta\left(\tau, N_{\mathbf{k} \lambda}\right)=\int_{b_{k} N_{\mathbf{k} \lambda}-\mathrm{i} \infty}^{b_{k} N_{\mathbf{k} \lambda}+\mathrm{i} \infty} \mathrm{e}^{\tau s} \Delta\left(s, N_{\mathbf{k} \lambda}\right) \frac{\mathrm{d} s}{2 \pi \mathrm{i}},
$$

where all the singularities are located in the left semiplane in respect to the line of integration. We can rewrite the last formula as follows [23]

$$
\Delta\left(\tau, N_{\mathbf{k} \lambda}\right)=\int_{-b_{k} N_{\mathbf{k} \lambda}}^{\infty} \mathrm{e}^{-\tau s}\left[\Delta\left(-s-\mathrm{i} 0, N_{\mathbf{k} \lambda}\right)-\Delta\left(-s+\mathrm{i} 0, N_{\mathbf{k} \lambda}\right)\right] \frac{\mathrm{d} s}{2 \pi \mathrm{i}} .
$$

We should mention that the expressions resulting from equations (25) and (43) are different. It signifies once again that the results obtained using FDT for the case 
of dissipations appear to be doubtful. Introducing energy broadenings into a theory assumes a certain approximation [33]. If we apply FDT out of its application field to the theory under these conditions, we use double approximation. This double approximation results in equation (25). Equation (43) makes it possible to calculate the correlation function $\left\langle\hat{\alpha}_{\mathbf{k} \lambda}^{+} \hat{\alpha}_{\mathbf{k} \lambda}\right\rangle$ avoiding such a double approximation.

Here, we will consider two cases, where the results can be simplified and the formulas are suitable for qualitative analysis. Let us consider the region of absorption. Let Doppler broadening be significant $\left|k-\varepsilon_{m}+\varepsilon_{\mu}\right| \approx \gamma_{\mathrm{D}}$. To compare the results with the formulas obtained using FDT and semiclassical theory of radiation under the temperatures $T \leq \varepsilon_{m}-\varepsilon_{\mu}$, we omit vacuum terms in equation (46). We consider a case of weak interaction which implies small $\mathscr{P}$. The maxima at $s=k N_{\mathbf{k} \lambda}$ of the subintegral functions of equation (48) are acute. It enables us to substitute $k=s / N_{\mathbf{k} \lambda}$ into the constructions $k\left(N_{\mathbf{k} \lambda} \pm 1\right)$ in denominators of equation (47). Now, we introduce a new variable $\omega=s / N_{\mathbf{k} \lambda}$. Equation (48) can be rewritten

$$
\begin{aligned}
& \Delta\left(\tau, N_{\mathbf{k} \lambda}\right)= \\
& =-\int_{-b}^{\infty} \mathrm{e}^{-\tau N_{\mathbf{k} \lambda} \omega}\left[\frac{1}{-\omega+k-\mathcal{P}^{\operatorname{scl}}(-\omega-\mathrm{i} 0)}-\frac{1}{-\omega+k-\mathcal{P}^{\operatorname{scl}}(-\omega+\mathrm{i} 0)}\right] \frac{\mathrm{d} \omega}{2 \pi \mathrm{i}},
\end{aligned}
$$

where

$$
\mathcal{P}^{\mathrm{scl}}(-\omega \pm \mathrm{i} 0)=-\sum_{m \mu \mathbf{p}}\left|\frac{P_{m \mu}^{\lambda}(k)}{\sqrt{2 k V}}\right|^{2}\left[\frac{\left\langle N_{m \mathbf{p}}\right\rangle}{\omega-\omega_{m \mu}-\frac{\mathbf{p k}}{M} \pm \mathrm{i} 0}+\frac{\left\langle N_{\mu \mathbf{p}}\right\rangle}{-\omega+\omega_{m \mu}+\frac{\mathbf{p k}}{M} \pm \mathrm{i} 0}\right] .
$$

The imaginary part of equation (50) depends upon the sum

$$
\left\langle N_{m \mathbf{p}}\right\rangle+\left\langle N_{\mu \mathbf{p}}\right\rangle
$$

Consequently, if the number of excited atoms is not negligible, it is impossible to express this function in terms of absorption coefficient resulting from (12).

To interpret equation (50), let us use the following equalities of dipole approximation

$$
P_{m \mu}^{\lambda}(\mathbf{k})=\mathrm{i} \omega_{m \mu} d_{m \mu}^{\lambda}(\mathbf{k}), \quad d_{m \mu}^{\lambda}(\mathbf{k})=\sum_{\nu} d_{m \mu}^{\nu} e_{\mathbf{k} \lambda}^{\nu}
$$

where $d_{m \mu}^{\nu}$ is a matrix element of dipole moment

$$
d_{m \mu}^{\nu}=\mathrm{e} \int \psi_{m}^{*} d^{\nu} \psi_{\mu} \mathrm{d} \mathbf{r}, \quad d^{\nu}=e r^{\nu}
$$

Let us introduce two different polarizabilities of atoms made up of Heisenberg operators of dipole moment $\breve{d}_{m \mu}^{\nu}(t)[32,34]$

$$
\alpha_{n}^{(c) \nu \nu^{\prime}}(\omega)=\mathrm{i} \int_{-\infty}^{\infty}\left\langle\hat{T} \check{d}^{\nu}(t) \check{d}^{\nu^{\prime}}(0)\right\rangle_{n} \mathrm{e}^{\mathrm{i} \omega t} \mathrm{~d} t=\sum_{n^{\prime}}\left(\frac{d_{n n^{\prime}}^{\nu} d_{n^{\prime} n}^{\nu^{\prime}}}{\omega_{n^{\prime} n}-\omega-\mathrm{i} 0}+\frac{d_{n n^{\prime}}^{\nu^{\prime}} d_{n^{\prime} n}^{\nu}}{\omega_{n^{\prime} n}+\omega-\mathrm{i} 0}\right),
$$


$\alpha_{n}^{(r) \nu \nu^{\prime}}(\omega)=\mathrm{i} \int_{-\infty}^{\infty}\left\langle\left[\check{d}^{\nu}(t) \check{d}^{\nu^{\prime}}(0)\right]\right\rangle_{n} \mathrm{e}^{\mathrm{i} \omega t} \mathrm{~d} t=\sum_{n^{\prime}}\left(\frac{d_{n n^{\prime}}^{\nu} d_{n^{\prime} n}^{\nu^{\prime}}}{\omega_{n^{\prime} n}-\omega-\mathrm{i} 0}+\frac{d_{n n^{\prime}}^{\nu^{\prime}} d_{n^{\prime} n}^{\nu}}{\omega_{n^{\prime} n}+\omega+\mathrm{i} 0}\right)$.

Here, $\hat{T}$ is the operator of chronological ordering. The retarding polarizability $\alpha_{n}^{(r) \nu \nu^{\prime}}(\omega)$ is an analytical function in the upper semiplane of complex $\omega$. It is the polarizability that determines the standard refractive index of macroscopic Maxwell equations. This refractive index has the same analytical properties as the retarding polarizability. The casualty polarizability $\alpha_{n}^{(c) \nu \nu^{\prime}}(\omega)$ determines the amplitude of photon scattering for the processes of coherent channel and Van der Vaals interaction of atoms [34]. But this tensor has a much wider field of application. It is the tensor that characterizes our polarization operator given by equation (46). Using equation (51), we can rewrite equation (50) in quasiresonance approximation

$$
\mathcal{P}^{\mathrm{scl}}\left(-\omega-\mathrm{i} 0 \mid 1_{\mathbf{k} \lambda}\right)=-\frac{\omega_{m \mu}}{2} \int\left(\sum_{\mu} n_{\mu} \alpha_{\mu}^{(c) \lambda \lambda}(\omega)+\sum_{m} n_{m} \alpha_{m}^{(c) \lambda \lambda}(\omega)\right) f(p) \frac{\mathrm{d} p}{2 \pi} .
$$

Here

$$
\begin{aligned}
& n_{\mu}=\frac{1}{V} \sum_{\mathbf{p}}\left\langle N_{\mu \mathbf{p}}\right\rangle, \quad n_{m}=\frac{1}{V} \sum_{\mathbf{p}}\left\langle N_{m \mathbf{p}}\right\rangle, \quad f(p)=\sqrt{\frac{2 \pi}{M T}} \exp \left(-\frac{p^{2}}{2 M T}\right), \\
& \alpha_{\mu}^{(c) \lambda \lambda}(\omega)=\alpha_{\mu}^{(r) \lambda \lambda}(\omega)=\sum_{m}\left(\frac{\left|d_{m \mu}^{\lambda}(\mathbf{k})\right|^{2}}{\omega_{m \mu}-\omega+\frac{p \omega}{M}-\mathrm{i} 0}\right), \\
& \alpha_{m}^{(c) \lambda \lambda}(\omega)=\sum_{\mu}\left(\frac{\left|d_{m \mu}^{\lambda}(\mathbf{k})\right|^{2}}{-\omega_{m \mu}+\omega-\frac{p \omega}{M}-\mathrm{i} 0}\right), \\
& \alpha_{m}^{(r) \lambda \lambda}(\omega)=\sum_{\mu}\left(\frac{\left|d_{m \mu}^{\lambda}(\mathbf{k})\right|^{2}}{-\omega_{m \mu}+\omega-\frac{p \omega}{M}+\mathrm{i} 0}\right) .
\end{aligned}
$$

Let us consider the excited media $\left(n_{m} \neq 0\right)$. For resonance region $\omega \approx \omega_{m \mu}$ the difference of sums

$$
\begin{aligned}
& n_{\mu} \alpha_{\mu}^{(r) \lambda \lambda}(\omega)+n_{m} \alpha_{m}^{(r) \lambda \lambda}(\omega) \propto n_{\mu}-n_{m} \\
& n_{\mu} \alpha_{\mu}^{(c) \lambda \lambda}(\omega)+n_{m} \alpha_{m}^{(c) \lambda \lambda}(\omega) \propto n_{\mu}+n_{m}
\end{aligned}
$$

is dramatic.

It is convenient to introduce the following terms. The retarding (standard or first) refractive index $n^{(r)}(\omega)$ is given by equation (12). The causal (or second) refractive index is given by

$$
n^{(c)}(\omega)=1-\frac{1}{\omega} \mathcal{P}^{\mathrm{scl}}\left(-\omega-\mathrm{i} 0 \mid 1_{\mathbf{k} \lambda}\right) .
$$


Let us substitute equation (49) into equation (43) and sum up over $N_{\mathbf{k} \lambda}$. Using equation (52), we get

$$
\left\langle\hat{\alpha}_{\mathbf{k} \lambda}^{+} \hat{\alpha}_{\mathbf{k} \lambda}\right\rangle=-\frac{1}{Z} \int_{-b_{k}}^{\infty} \frac{\mathrm{e}^{-\frac{\omega}{T}}}{\left(1-\mathrm{e}^{-\frac{\omega}{T}}\right)^{2}}\left[\frac{1}{\omega n^{(c)}(\omega)-k}-\frac{1}{\omega n^{(c) *}(\omega)-k}\right] \frac{\mathrm{d} \omega}{2 \pi \mathrm{i}} .
$$

The fact that $\left\langle N_{\mathbf{k} \lambda}^{m}\right\rangle$ should be finite for any $m>0$ signifies that small frequencies contribute little to the integral equation (53). Consequently, we assume that the constant $b_{k}$ is defined by the left minimum of the subintegral function for $\omega>0$. To calculate the normalizing constant $Z$, we use the following formula [23]

$$
-\frac{1}{Z} \sum_{N_{\mathbf{k} \lambda}=1}^{\infty} \Delta\left(\frac{1}{T}, N_{\mathbf{k} \lambda}\right)=1
$$

Now,

$$
Z=-\int_{-b_{k}}^{\infty} \frac{1}{1-\mathrm{e}^{-\frac{\omega}{T}}}\left[\frac{1}{\omega n^{(c)}(\omega)-k}-\frac{1}{\omega n^{(c) *}(\omega)-k}\right] \frac{\mathrm{d} \omega}{2 \pi \mathrm{i}} .
$$

We have shown that the single-time correlation function does not depend upon the standard refractive index $n^{(r)}(\omega)$ but depends upon the causal one $n^{(c)}(\omega)$. There is no $n^{(c)}(\omega)$ in phenomenological theory, which operates only with the standard refractive index. If we put $n^{(c)}(\omega)=1$ in equations (53) and (54), we get the result

$$
\left\langle\hat{\alpha}_{\mathbf{k} \lambda}^{+} \hat{\alpha}\right\rangle=\frac{1}{\exp \frac{k}{T}-1},
$$

offered by Klimontovich [19]. In this case, the single-time correlation function does not contain relaxation constants at all.

Now, we can obtain the final results using equations (11)-(14)

$$
\begin{aligned}
\left\langle\check{\alpha}_{\mathbf{k} \lambda}^{+}(t) \check{\alpha}_{\mathbf{k} \lambda}\left(t^{\prime}\right)\right\rangle_{E} & =\mathrm{i}\left\langle\hat{\alpha}_{\mathbf{k} \lambda}^{+} \hat{\alpha}_{\mathbf{k} \lambda}\right\rangle\left(\frac{1}{E n^{(r) *}(-E)+k}-\frac{1}{E n^{(r)}(-E)+k}\right), \\
\left\langle\check{\alpha}_{\mathbf{k} \lambda}(t) \check{\alpha}_{\mathbf{k} \lambda}^{+}\left(t^{\prime}\right)\right\rangle_{E} & =\mathrm{i}\left\langle\hat{\alpha}_{\mathbf{k} \lambda} \hat{\alpha}_{\mathbf{k} \lambda}^{+}\right\rangle\left(\frac{1}{E n^{(r)}(E)-k}-\frac{1}{E n^{(r) *}(E)-k}\right) .
\end{aligned}
$$

Equations (55) and (56) are sufficient to write down the result for correlation function (8) we are seeking. We would like to emphasize that this correlation function is described by two different refractive indexes, as it follows from (53)-(56). Consequently, it cannot be derived using the methods of classical physics. In the region under consideration, $n_{m}$ and $n_{\mu}$ are comparable. It results in violation of equation (4) in this region.

Let us consider a quantum region $k \sim \omega_{m \mu} \gg T$. In equation (43), the only term with $N_{\mathbf{k} \lambda}=1$ is significant. Here, the vacuum term in $\mathscr{P}\left(s, N_{\mathbf{k} \lambda}\right)$ is not negligible

$$
\begin{aligned}
\mathcal{P} & \left(-\omega-\mathrm{i} 0 \mid 1_{\mathbf{k} \lambda}\right)= \\
& =-\sum_{m \mu \mathbf{p}}\left|\frac{P_{m \mu}^{\lambda}(k)}{\sqrt{2 k V}}\right|^{2}\left[\frac{2 N_{m}(\mathbf{p})}{-\omega-\omega_{m \mu}+2 k-\frac{\mathbf{p k}}{M}-\mathrm{i} 0}+\frac{N_{\mu}(\mathbf{p})}{-\omega+\omega_{m \mu}+\frac{\mathbf{p k}}{M}-\mathrm{i} 0}\right] .
\end{aligned}
$$


The result cannot be represented even in terms of $n^{(c)}(\omega)$.

The final result depending upon two different refractive indexes cannot be reduced to equation (4). If the contribution of $n_{m}$ is negligible in comparison with $n_{\mu}$, the final result depends only upon $n^{(r)}(E)$. In this case we get equation (28) instead of equation (4) resulting from FDT. These formulas are discussed in section 4.

Thus, equation (4) incorrectly describes the region of absorption of media with $n_{m} \neq 0$.

Using equations (27), (53), (55) and (56) after inverse Fourier transformation, we come to the conclusion that the single-time correlation function of vector potentials depends upon the causal refractive index $n^{(c)}(\omega)$ and does not depend on $n^{(r)}(\omega)$.

Let us consider the regions of transparency, where $\left|k-\varepsilon_{m}+\varepsilon_{\mu}\right| \gg \gamma_{\mathrm{D}}$. We may neglect Doppler terms $\sim$ pk in equation (47). Now, we can calculate all the integrals explicitly [23]. For an extra assumption

$$
\eta_{\mu}=\frac{c}{\left|k-\omega_{m \mu}\right|^{2}} \ll 1, \quad \eta_{m}=\frac{a}{\left|k-\omega_{m \mu}\right|^{2}} \ll 1
$$

where

$$
c=\sum_{m \mu}\left|P_{m \mu}^{\lambda}\right|^{2}\left\langle N_{\mu}\right\rangle, \quad a=\sum_{m \mu}\left|P_{m \mu}^{\lambda}\right|^{2}\left\langle N_{m}\right\rangle, \quad\left\langle N_{m, \mu}\right\rangle=\int\left\langle N_{m, \mu}(\mathbf{p})\right\rangle \frac{\mathrm{d} \mathbf{p}}{(2 \pi)^{3}}
$$

we get [23]

$$
\begin{aligned}
\left\langle\hat{\alpha}_{\mathbf{k} \lambda}^{+} \hat{\alpha}_{\mathbf{k} \lambda}\right\rangle & = \\
=\{ & \left.\exp \left(\frac{k+\left(n_{\mu}-\eta_{m}\right)\left(k-\omega_{m \mu}\right)}{T}\right)-1\right\}^{-1}\left[1+\eta_{\mu} \frac{1-\exp \left[\left(-\left(\omega_{m \mu}-k\right) / T\right]\right.}{\exp (k / T)-1}\right. \\
& \left.+\eta_{m}\left(1-\exp -\frac{k-\omega_{m \mu}}{T}\right)\left(1+\frac{1}{\exp (k / T)-1}\right)\right] \\
& -\frac{\eta_{\mu}}{\exp (k / T)-1}\left(1+\frac{2}{\exp (k / T)-1}\right)\left(1-\exp \left[-\frac{\omega_{m \mu}-k}{T}\right]\right) \\
& -\frac{2 \eta_{m}}{\exp (k / T)-1}\left(1+\frac{1}{\exp (k / T)-1}\right)\left(1-\exp \left[-\frac{k-\omega_{m \mu}}{T}\right]\right)
\end{aligned}
$$

If we used a semiclassical theory with standard reflective index resulting in equation (2), we would obtain only the first factor of equation (57) taken in braces. Thus, our using the semiclassical theory results in a loss of terms of the same order of magnitude as the ones accounted for.

Therefore, equations (2) and (4) are not perfect even for the case of transparency regions. In the transparency regions, the terms $k\left(N_{\mathbf{k} \lambda} \pm 1\right)$ of equation (47) are significant. Their physical meaning is discussed in the introduction. For transparent regions, these terms result in splitting of optical spectrum into three branches [23]. All the branches considerably contribute to equation (57). This situation is not taken into account in equation (2). 


\section{Conclusions}

A theory of thermodynamic fluctuations of electromagnetic field in slightly absorbing media of two-level atoms is developed in microscopic framework without fluctuation-dissipation theorem.

The case of excited media with relaxation broadenings is considered. The results obtained here contradict those obtained using the FDT. The combined application of FDT and relaxation broadening approximation, as we show, results in contradictory formulas.

It is shown that the Klimontovich hypothesis, which states that single-time correlation functions do not depend on relaxation constants under the condition of thermodynamic equilibrium, should be modified.

Our analysis is carried out using the $\Gamma$-operator method. This method does not depend on FDT. The method has an advantage that makes it possible to account for all orders of photon-photon correlation functions. As a result, we show that it is impossible to describe the field fluctuations with the standard refractive index only.

We carried out the analysis without phenomenological hypotheses. It is shown that quantum effects of electromagnetic field should not be omitted if the atoms are considered as quantum objects.

Here, we consider a two-level model of atom with Zeeman sublevels. Thus, the passage to the limit $\hbar \rightarrow 0$ is not permitted.

We show that the quantum field effects affect the correlation functions of electromagnetic field as follows.

1. Let the concentration of excited atoms $n_{m}$ be comparable with the concentration of the ground state ones $n_{\mu}$. The process of photon scattering on an excited atom is described as follows. First, a photon is emitted by an atom and only then the incident photon is absorbed. There are two photons in a virtual state. Consequently, the dispersion relation is complicated. The process of scattering is not local. It results in standard theory violation in the region of absorption $\left|k-\varepsilon_{m}+\varepsilon_{\mu}\right| \sim \gamma_{\mathrm{D}}$. We show that in this region it is impossible to express the fluctuations of electromagnetic field in terms of the standard refractive index only. We introduce the second refractive index $n^{(c)}(\omega)$ into the theory. If we put $n^{(c)}(\omega)=1$ in phenomenological theory, we get the result offered by Klimontovich for the single-time correlation function. Here, we should mention the following thing. Since, in accordance with FDT, all the correlation functions (including the single-time ones) are determined by the Green functions of the system, the quantum corrections of higher order for the Green functions are expected to depend upon $n^{(c)}(\omega)$. This problem should be investigated in subsequent works.

2. Let the concentration of excited atoms be negligible $n_{m} \ll n_{\mu}$. It is possible only in quantum region $\varepsilon_{\mu}-\varepsilon_{m}>T$. Here, it is possible to express the results in terms of the standard refractive index even in the region of absorption. But 
instead of standard equation (4) and equivalent equation (17), we should use equation (28).

3. Let $\left|k-\varepsilon_{m}+\varepsilon_{\mu}\right| \gg \gamma_{\mathrm{D}}$ (region of transparency). The following effect of photon correlation is significant here. Let one of $N_{\mathbf{k} \lambda}$ photons of a $(\mathbf{k}, \lambda)$ mode be scattered. Then, the other $N_{\mathbf{k} \lambda}-1$ photons should be taken into account since all the photons are described with a single wave function. The correlation effects result in photon spectrum splitting and violate the results of standard theory as well as the Clausius formula (2).

We show that a theory of thermodynamic fluctuations of electromagnetic field including complex refractive index should be developed without FDT. The theory, as it is shown, requires a correct accounting for photon-photon correlation functions. Thus, the theory of the correlation function $\left\langle\mathscr{E} \nu(\mathbf{r}, t) \mathscr{E} \nu^{\prime}\left(\mathbf{r}^{\prime}, t^{\prime}\right)\right\rangle$ offered here is free of logical drawbacks both in regions of absorption and transparency.

We would like to acknowledge A.A. Rukhadze and the participants of the seminar of theoretical department of General Physics Institute RAS, where this work was discussed.

\section{References}

1. Rytov S.M. Theory of Electric Fluctuations and Thermal Radiation. Moscow, Nauka, 1953 (in Russian).

2. Lifshitz E.M., Pitaevski L.P. Statistical Physics, part 2. Oxford, ButterworthHeinenmann, 1980.

3. Planck M. // Ann. D. Physik, 1901, vol. 4, p. 553.

4. Nyquist H. // Phys. Rev., 1928, vol. 32, p. 110.

5. Levin M.L., Rytov S.M. Theory of Equilibrium Thermal Fluctuations in Electrodynamics. Moscow, Nauka, 1967 (in Russian).

6. Rytov S.M. // Doklady Akademii Nauk SSSR, 1952, vol. 87, p. 535 (in Russian).

7. Langevin P. // C. R., 1908, vol. 146, p. 530.

8. Leontovich M.A., Rytov S.M. // Zh. Eksp. Teor. Fiz., 1952, vol. 23, p. 246.

9. Zemtsov Yu.K., Sechin A.Yu., Starostin A.N. // JETP, 1998, vol. 87, p. 76.

10. Lifshitz E.M. // Doklady Akademii Nauk SSSR, 1954, vol. 97, p. 643 (in Russian).

11. Lifshitz E.M. // Sov. Phys. JETP, 1956, vol. 2, p. 73.

12. Casimir H.B.C., Polder D. // Phys. Rev., 1948, vol. 73, p. 360.

13. Callen H.B., Welton T.A. // Phys. Rev., 1951, vol. 83, p. 34.

14. Callen H.B., Barash M.L., Jackson J.L. // Phys. Rev., 1952, vol. 88, p. 1382.

15. Landau L.D., Lifshitz E.M. Electrodynamics of Continuous Media. Oxford, Pergamon, 1960.

16. Rytov S.M. // Sov. Phys. Doklady, 1956, vol. 1, p. 555.

17. Rytov S.M., Kravtsov Yu.A., Tatarskii V.I. Principles of Statistical Radiophysics. Berlin-London, Springer-Verlag, 1987.

18. Barash Yu.S. Van der Vaals forces. Nauka, Moscow, 1988 (in Russian).

19. Klimontovich Yu.L. // Sov. Phys. Usp., 1987, vol. 30, p. 154.

20. Heitler W. The Quantum Theory of Radiation. Claredon Press, Oxford, 1954. 
21. Akhiezer A.I., Berestetskii V.B. Quantum Electrodynamics. New York, Interscience Publishers, 1965.

22. Abrikosov A.A., Gorkov L.P., Dzyaloshinski I.E. Methods of Quantum Field Theory in Statistical Physics. New York, Dover Publications, 1975.

23. Veklenko B.A. // JETP, 1998, vol. 87, p. 270.

24. Veklenko B.A. // Sov. Phys. JETP, 1989, vol. 69, p. 258.

25. Veklenko B.A. // JETP, 2001, vol. 92, p. 940.

26. Tatarskii V.I. // Sov. Phys. Usp., 1987, vol. 30, p. 134.

27. Bogoliubov N.N., Tyablikov S.V. // Sov. Phys. Doklady, 1959, vol. 4, p. 589.

28. Shiktorov P., Starikov E., Gruzinskis V., Reggiani L. - In: Proc. of the $17^{\text {th }}$ Int. Conf. on Noise and fluctuations. Prague, August 2003, ed. J. Sikula, p. 31.

29. Ford C.W., O'Connell R.F. // Phys. Rev. Lett, 1996, vol. 77, p. 798.

30. Crobert H. // Z. Phys. B, 1982, vol. 49, p. 161.

31. Talkner P. // Ann. Phys. (USA), 1986, vol. 167, p. 390.

32. Veklenko B.A., Sherkunov Yu.B. // Izv. Vuzov. 2000, vol. 66, p. 17 (in Russian).

33. Ginzburg V.L., Pitaevskii L.P. // Sov. Phys. Usp., 1987, vol. 30, p. 168.

34. Berestetski V.B., Lifshitz E.M., Pitaevski L.P. Quantum Electrodynamics. Oxford, Pergamon, 1982.

\title{
Термодинамічні флуктуації електромагнітного поля в слабо адсорбуючому середовищі
}

\author{
Б.А.Векленко ${ }^{1}$, Ю.Б.Шеркунов ${ }^{2}$ \\ 1 Інститут високих температур РАН, 127412, Москва, Росія \\ 2 Інститут теплофізики екстремальних станів Об'єднаного інституту \\ високих температур РАН, 127412, Москва, Росія
}

Отримано 26 грудня 2003 р., в остаточному вигляді 10 березня 2004 p.

Розвинуто теорію термодинамічних флуктуацій електромагнітного поля в слабо адсорбуючому середовищі на основі квантової електродинаміки (метод Г -операторів) без використання феноменології. Розглядається гіпотеза, запропонована Ю.Л. Климонтовичем. Показана необхідність коректного врахуваня фотон-фотонної кореляційної функції. Результати порівнюються з отриманими в рамках стандартної теорії, що грунтується на флуктуаційно-дисипаційній теоремі. Показано, що результати стандартної теорії не можуть бути застосованими, принаймні, у випадку термічно активованого середовища атомів, що описуються двохрівневою моделлю.

Ключові слова: термодинамічні флуктуації, флуктуаційно-дисипативна теорема, кореляційна функція, електромагнітні поля в слабо поглинаючих середовищах

PACS: $42.50 . c t$ 